\title{
Generalized interfacial energy and size effects in composites
}

\author{
George Chatzigeorgiou ${ }^{a}$, Fodil Meraghni ${ }^{\mathrm{a}}$, Ali Javili ${ }^{\mathrm{b}, *}$ \\ a LEM3-UMR 7239 CNRS, Arts et Métiers ParisTech Metz, 4 Rue Augustin Fresnel Metz 57078, France \\ ${ }^{\mathrm{b}}$ Department of Mechanical Engineering, Bilkent University, Ankara 06800, Turkey
}

\section{A R T I C L E I N F O}

\section{Article history:}

Received 24 April 2017

Revised 6 June 2017

Accepted 6 June 2017

Available online 8 June 2017

\section{Keywords:}

General interface

Ultimate size effect

Nano-composites

Capillary effect

CCA

\begin{abstract}
A B S T R A C T
The objective of this contribution is to explain the size effect in composites due to the interfacial energy between the constituents of the underlying microstructure. The generalized interface energy accounts for both jumps of the deformation as well as the stress across the interface. The cohesive zone and elastic interface are only two limit cases of the general interface model. A closed form analytical solution is derived to compute the effective interface-enhanced material response. Our novel analytical solution is in excellent agreement with the numerical results obtained from the finite element method for a broad variety of parameters and dimensions. A remarkable observation is that the notion of size effect is theoretically bounded verified by numerical examples. Thus, the gain or loss via reducing the dimensions of the microstructure is limited to certain ultimate values, immediately relevant for designing nano-composites.
\end{abstract}

(c) 2017 Elsevier Ltd. All rights reserved.

\section{Introduction}

Homogenization (Hill, 1963; 1972; Ogden, 1974) is a commonly accepted methodology to explain the overall response of composite materials based on its constituents at the microscale. While the classical homogenization is well-established today, the influence of interfaces at the microscale remains elusive and poorly understood. This contribution investigates on the impact of interfaces at the microscale on the effective material response through an interface-enhanced homogenization scheme from both analytical and numerical perspectives.

The interphases between various constituents of a heterogeneous microstructure can play a crucial role on the overall material response. The general interface model here represents the finite thickness interphase. Note, the idea of the general interface model follows the seminal work of Hashin (2002) where he distinguishes between perfect and imperfect interface models. Furthermore, McBride et al. (2012) show that classical interface models cannot capture the response of heterogeneous material layers, see Fig. 1. Emerging applications of nano-materials require better understanding of interfaces since the influence of lower-dimensional media on the overall material response increases with decreasing size.

Interfaces can be categorized into four models according to their kinematic or kinetic characteristics, as shown in Fig. 2. The perfect interface model does not allow for the displacement jump nor the traction jump across the interface. The elastic interface model is semi-imperfect in the sense that it is kinematically coherent but kinetically non-coherent. Interface elasticity theory (Daher and Maugin, 1986; Dell'Isola and Romano, 1987; Fried and Gurtin, 2007; Gurtin and Murdoch, 1975; Moeckel, 1975; Murdoch, 1976) endows the interface with an elastic resistance along the interface. The traction jump across the interface is due to the interface stress (see Chen et al., 2006; Javili et al., 2013c, among others). Interface and surface

\footnotetext{
* Corresponding author.

E-mail addresses: georges.chatzigeorgiou@ensam.eu (G. Chatzigeorgiou), fodil.meraghni@ensam.eu (F. Meraghni), ajavili@bilkent.edu.tr (A. Javili).
} 


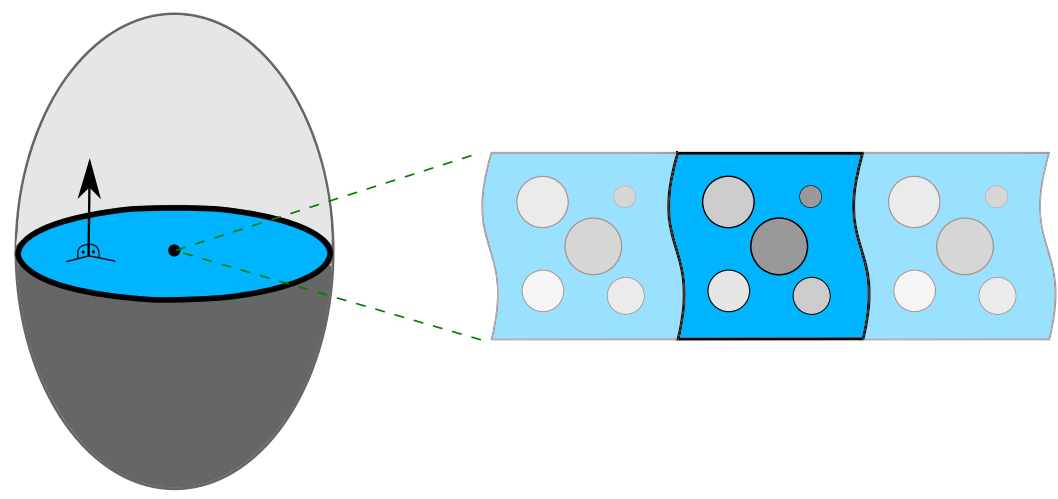

Fig. 1. Motivation for the need of a general interface model. Homogenization of heterogeneous material layers shows that even the simplest elastic response requires a general interface model to be properly captured McBride et al. (2012). The zero-thickness interface model (left) is representative of a finite thickness interphase (right).
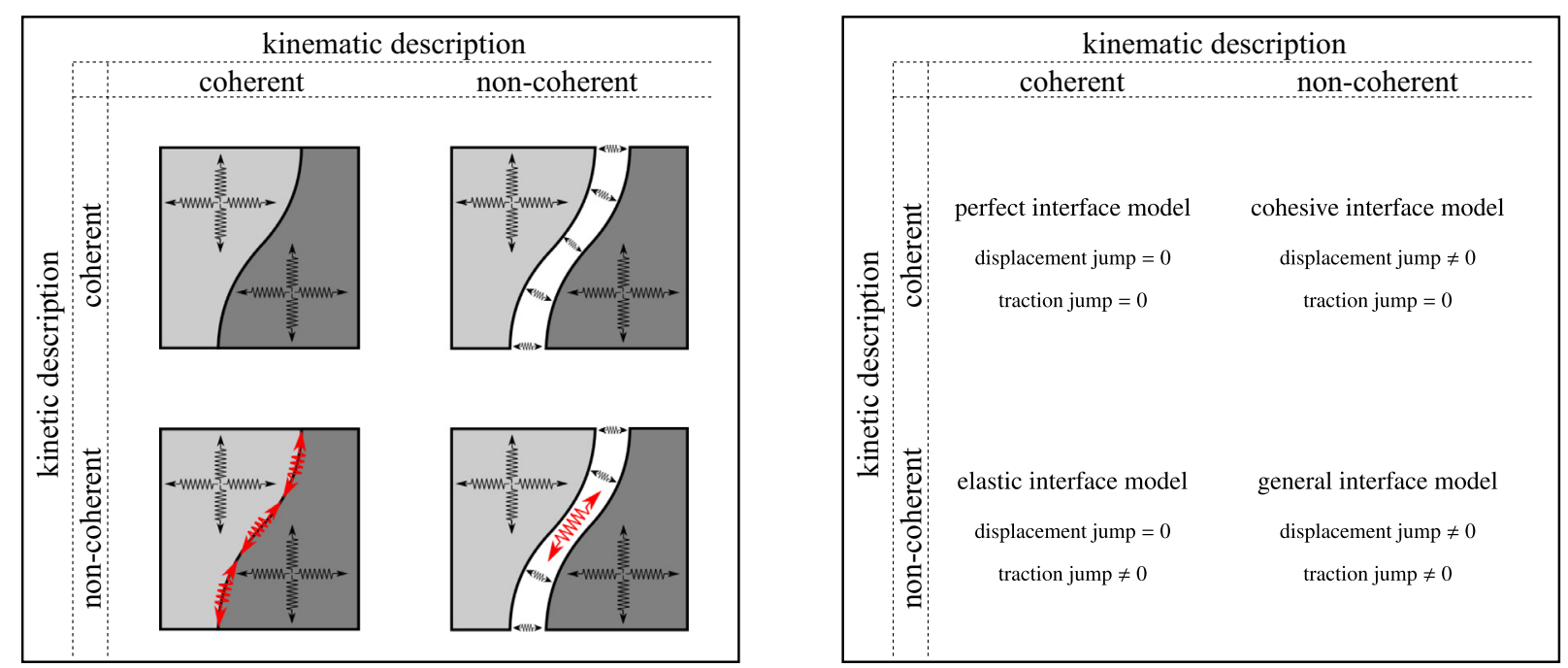

Fig. 2. An overview of interface models. Graphical illustration (left) and mathematical explanation (right). From the viewpoint of continuum mechanics, interfaces can be divided into four categories depending on their kinematic or kinetic characteristics. The elastic interface model does not allow for the displacement jump, but the traction may suffer a jump across the interface. The cohesive interface model on the contrary, allows for the displacement jump but assumes the continuity of the traction field across the interface. The intersection of elastic and cohesive interface model is the perfect interface model for which both the traction and displacement across the interface are continuous. This contribution formulates the general interface model which encompasses all other interface types.

elasticity theory is a mature field investigated in Altenbach and Eremeyev (2011); Chhapadia et al. (2011); Cordero et al. (2016); Dingreville et al. (2014); Dingreville and Qu (2008); Duan et al. (2009); Fried and Todres (2005); Gao et al. (2014); Gurtin et al. (1998); Huang and Wang (2006); Javili et al. (2013a); Liu et al. (2017); Steigmann and Ogden (1999); Steinmann (2008); Wang et al. (2010a); 2010b) among others. ${ }^{1}$ The cohesive interface model allows for the displacement jump across the interface, but remains kinetically coherent hence, semi-imperfect. The cohesive interface model dates back to the seminal works (Barenblatt, 1959; 1962; Dugdale, 1960) and has been extensively studied (Alfano and Crisfield, 2001; van den Bosch et al., 2006; Charlotte et al., 2006; Despringre et al., 2016; Dimitri et al., 2015; Fagerstr and Larsson, 2006; Gasser and Holzapfel, 2003; Mosler and Scheider, 2011; Ortiz and Pandolfi, 1999; Park and Paulino, 2013; Park et al., 2009; Qian et al., 2017; Tijssens et al., 2000; Wu et al., 2016; Xu and Needleman, 1994) in the past. The perfect interface model is the intersection of the two semi-perfect interface models. From the perspective of deriving interface models as asymptotic limits of thin interphases, the cohesive interface model is derived as the limit case of soft interphases and is termed spring

\footnotetext{
1 An important Erratum Huang and Wang (2010) on Huang and Wang (2006) was later published by its authors. Furthermore, the same authors published a more comprehensive version of their work on interfacial energy and micromechanics with interface effect in Huang and Wang (2013).
} 
interface model. On the contrary, the elastic interface model is obtained as the limit case of stiff interphases and is termed stress interface model, see also Wang et al. (2005). The general (imperfect) interface model unifies all various types of interfaces and is both kinematically and kinetically non-coherent. The general interface model (Hashin, 2002) has been studied in Benveniste (2006); 2013b); Benveniste and Miloh (2001); Benveniste and Milton (2010a); 2010b); Bö (1994); Gu and He (2011); Gu et al. (2011); Monchiet and Bonnet (2010), but derived from simplified asymptotic limits of thin interphases. Particulate composites with general interfaces have been examined in Gu et al. (2014). As illustrated in Fig. 1, the appropriate interface model to describe the heterogeneous material layer between the microstructures depends on the underlying microstructure of the interfacial layer. For instance, if the microstructure acts similar to a set of parallel springs normal to the interface, the cohesive interface model is suitable. On the other hand, if the interfacial layer shows extremely high resistance against opening, then the elastic interface model can capture the resistance against deformations along the interface. Obviously, both the elastic interface model as well as the cohesive interface model are the limits of a generalized interface model accounting for both in-plane and out-of-plane resistance of the interface.

Homogenization pioneered by Hill and Ogden, is extended in this contribution to account for the interfaces at the microscale. For further details on homogenization, see the reviews Geers et al. (2010); Kanout et al. (2009); Matous et al. (2017); Mura et al. (1996); Ostoja-Starzewski et al. (2016); Saeb et al. (2016) and references therein. Although the classical first-order homogenization is well-established today, it suffers from the lack of a length-scale. In particular, the classical homogenization cannot capture the size effect in the material response. Accounting for interfaces naturally leads to a scale dependent overall response since the area-to-volume ratio is proportional to the inverse of the dimension. Scale dependent macroscopic behavior due to lower-dimensional elasticity at the microscale has been a popular topic in the past decade from both analytical (Brisard et al., 2010; Chatzigeorgiou et al., 2015; Duan and Karihaloo, 2007; Duan et al., 2005b; Huang and Sun, 2007; Li et al., 2011; Lim et al., 2006; Mogilevskaya et al., 2008; Nazarenko et al., 2017; Sharma, 2004; Sharma et al., 2003; Sharma and Wheeler, 2007; Tian and Rajapakse, 2007) and computational perspectives (Fritzen and Leuschner, 2015; Javili et al., 2015; 2013b; Monteiro et al., 2011; Tu and Pindera, 2014; Yvonnet et al., 2008). Furthermore, it can be argued that the size effect due to the interface elasticity is physically meaningful supported by atomistic simulations (Davydov et al., 2013; Elsner et al., 2017; He and Lilley, 2008; Levitas and Samani, 2011; Olsson and Park, 2012; Park and Klein, 2007; 2008; Park et al., 2006; Yvonnet et al., 2012). ${ }^{2}$ The aforementioned contributions deal only with the size effect due to either the elastic interface model or the cohesive interface model. This contribution elaborates on the size effect due to the general interface model. Obviously, the current model reduces to both the cohesive and elastic interface models.

The classification of the interface models here are based on their "kinematic" descriptions. More precisely, the intrinsic feature of the elastic interface model is that it is geometrically coherent in the sense that the displacement jump across the interface vanishes. On the contrary, the cohesive interface model is geometrically non-coherent and allows for the displacement jump. The constitutive response of each interface model remains to be discussed and depends on its energy arguments. For instance, the energy of the elastic interface model may depend on the interface strain, curvature or higher gradients of the interface strain. The interface elastic energy in this contribution is assumed to be only a function of the interface strain and not its gradients nor the curvature. It can be argued that for certain applications in biomembranes and lipid bilayers the curvature dependence of the interface may not be negligible, see e.g. Gao et al. (2017). Accounting for the curvature formally fits in the framework developed here, but it also leads to the dependence of the overall response on additional interface parameters. Note, the ultimate goal of this contribution is to carry out an interface parameter identification via analyzing the size dependent overall response of the material. Introducing more complicated interface models introduces more parameters without necessarily providing additional insight. Eventually, one can make the interface model so complicated such that no analytical solution for the overall response can be provided, but that completely defeats the initial purpose of this manuscript. Here, for the first time we provide an analytical form for the effective behavior of a heterogeneous material containing generalized interfaces.

\subsection{Organization of this manuscript}

This manuscript is organized as follows. Notation and definitions are shortly introduced and key contributions of this manuscript are highlighted immediately afterward. Section 2 defines the problem of interest and briefly formulates the associated governing equations. Balance equations of general interfaces are given in Section 2.1. Section 2.2 elaborates on the micro-to-macro transition of continua accounting for general interfaces. Analytical solutions for effective material properties of unidirectional fiber composites are obtained via the composite cylinder approach in Section 3. Section 4 investigates the influence of general interfaces on the overall behavior of materials via a series of parametric studies. In particular, the proposed semi-analytical solution is compared against numerical results using the finite element method. Furthermore, the size effect due to interfaces is demonstrated and the notion of ultimate size effect is discussed. Section 5 concludes this work and discusses possible extensions and outlooks.

\footnotetext{
${ }^{2}$ Alternatively, a second-order homogenization scheme can be developed (Kouznetsova et al., 2004; 2002) to introduce a length-scale into homogeniza-
} tion. 


\subsection{Notations and definitions}

Quantities defined on the interface are distinguished from those in the bulk by a bar placed above the quantity. That is, $\{\bar{\bullet}\}$ refers to an interface variable with its bulk counterpart being $\{\cdot\}$. Analogously, surface and curve quantities are denoted as $\{\hat{\bullet}\}$ and $\{\tilde{\bullet}\}$, respectively to be distinguished from the bulk quantity $\{\bullet\}$. Moreover, macroscale quantities are differentiated from microscale quantities by the left super-script "M" placed next to the quantity. That is, M\{ $\bullet\}$ refers to a macroscopic variable with its microscopic counterpart being $\{\bullet\}$. Direct notation is adopted throughout. The dyadic product of two vectors $\boldsymbol{a}$ and $\boldsymbol{b}$ is a second-order tensor $\boldsymbol{D}=\boldsymbol{a} \otimes \boldsymbol{b}$ with $[\boldsymbol{D}]_{i j}=[\boldsymbol{a}]_{i}[\boldsymbol{b}]_{j}$. The scalar product of two vectors $\boldsymbol{a}$ and $\boldsymbol{b}$ is denoted $\boldsymbol{a} \cdot \boldsymbol{b}=[\boldsymbol{a}]_{i}[\boldsymbol{b}]_{i}=[\boldsymbol{a} \otimes \boldsymbol{b}]: \boldsymbol{i}$ where $\boldsymbol{i}$ is the second-order identity tensor. The scalar product of two second-order tensors $\boldsymbol{A}$ and $\boldsymbol{B}$ is denoted $\boldsymbol{A}: \boldsymbol{B}=[\boldsymbol{A}]_{i j}[\boldsymbol{B}]_{i j}$. The composition of two second-order tensors $\boldsymbol{A}$ and $\boldsymbol{B}$, denoted $\boldsymbol{A} \cdot \boldsymbol{B}$, is a second-order tensor with components $[\boldsymbol{A} \cdot \boldsymbol{B}]_{i j}=[\boldsymbol{A}]_{i m}[\boldsymbol{B}]_{m j}$. The action of a second-order tensor $\boldsymbol{A}$ on a vector $\boldsymbol{a}$ is given by $[\boldsymbol{A} \cdot \boldsymbol{a}]_{i}=[\boldsymbol{A}]_{i j}[\boldsymbol{a}]_{j}$. The average and jump of a quantity $\{\bullet\}$ over the interface are defined by $\{\{\bullet \bullet\}\}=\frac{1}{2}\left[\{\bullet\}^{+}+\{\bullet\}^{-}\right]$and $\llbracket\{\bullet\} \rrbracket=\{\bullet\}^{+}-\{\bullet\}^{-}$, respectively. The average and jump operators show the property $\llbracket\{\bullet\} \cdot\{0\} \rrbracket=\llbracket\{\bullet\} \rrbracket \cdot\{\{\{0\}\}\}+\{\{\bullet \bullet\}\} \cdot \llbracket\{0\} \rrbracket$.

Here, unlike the classical first-order homogenization, the term "size" refers to the actual dimension of the representative volume element (RVE). See for instance Khisaeva and Ostoja-Starzewski (2006) for further details on the definition of the "size" of the RVE within the classical first-order homogenization context. For the sake of simplicity, this contribution deals with perfectly periodic microstructures such that the unit cell is by definition representative and hence a RVE.

\subsection{Key aspects and contributions}

The main objective of this contribution is to explain the size effect in composites due to the interfacial energy of the underlying microstructure. The key features and contributions of this manuscript are

- to categorize interface models and propose a generalized model,

- to formulate generalized interfaces within a small-strain continuum mechanics setting,

- to incorporate the general interface model into a micro-to-macro transition framework,

- to derive an analytical solution for the effective properties of composites containing general interfaces,

- to compare the novel analytical solution with numerical results in a series of parametric studies,

- to analyze the size effect due to interfaces embedded within the microstructure,

- to introduce the notion of ultimate size effect and optimal RVE size.

\section{Governing equations}

Consider a continuum body that occupies the configuration $\mathrm{M}_{\mathcal{B}}$ at the macroscale as shown in Fig. 3. The configuration ${ }^{M_{\mathcal{B}}}$ is heterogeneous and composed of its RVE at the microscale $\mathcal{B}$. At the microscale, the interphase zone between the inclusion and the matrix is captured by the zero-thickness general interface model. The constitutive behavior of the components at the microscale is assumed to be known and collectively resulting in the macroscale response. That is, prescribing the macro strain ${ }^{\mathrm{M}} \boldsymbol{\varepsilon}$ on the RVE results in the macro stress ${ }^{\mathrm{M}} \boldsymbol{\sigma}$. Homogenization provides a framework to appropriately relate the micro quantities to macro quantities. In homogenization of heterogeneous media, an underlying assumption is the separation of

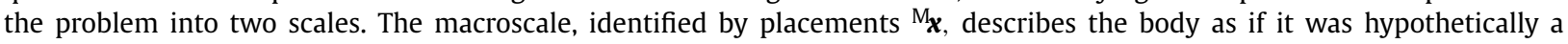
homogeneous medium. The microscale, identified by placements $\boldsymbol{x}$, describes the microstructure of the composite. Homogenization requires (i) the microscale to be significantly smaller than the macroscale and (ii) an existing RVE with sufficient details of the microstructure such that it can describe the overall behavior of the composite in an average sense. The forthcoming discussions in this section follow closely the recent contributions of the authors (Chatzigeorgiou et al., 2015; Javili et al., 2017).

\subsection{Generalized interfaces}

The purpose of this section is to establish the balance equations governing continua embedding general interfaces. These governing equations obviously correspond to the microscale in Fig. 3. The interface $\mathcal{I}$ splits the body $\mathcal{B}$ into two disjoint subdomains $\mathcal{B}^{-}$and $\mathcal{B}^{+}$. The interface unit normal $\overline{\boldsymbol{n}}$ points from the minus to the plus side of the interface. The outward unit normal to the external boundary $\mathcal{S}$ is denoted $\widehat{\boldsymbol{n}}$. If the interface is entirely enclosed within the body, its intersection with the external surface $\mathcal{S}$ is an empty set. However, if the interface is open and reaches the boundary of the microscale, its intersection with the external surface $\mathcal{S}$ is the curve $\mathcal{C}$ as

$$
\mathcal{I} \cap \mathcal{S}=\emptyset \Leftrightarrow \text { closed interface and } \mathcal{I} \cap \mathcal{S}=\mathcal{C} \quad \Leftrightarrow \quad \text { open interface . }
$$

The outward unit normal to the curve $\mathcal{C}$ but tangent to the interface $\mathcal{I}$ is denoted $\tilde{\boldsymbol{n}}$ and plays an important role to derive the balance equations for open interfaces.

The displacement in the body is denoted $\boldsymbol{u}$. To proceed, it proves convenient to define the minus and plus sides of the interface as $\mathcal{I}^{-}=\mathcal{I} \cap \partial \mathcal{B}^{-}$and $\mathcal{I}^{+}=\mathcal{I} \cap \partial \mathcal{B}^{+}$, respectively. The displacement on the minus and plus sides of the interface are denoted $\boldsymbol{u}^{-}$and $\boldsymbol{u}^{+}$, respectively. The displacement jump across the interface $\llbracket \boldsymbol{u} \rrbracket$ needs not vanish for the general interface 


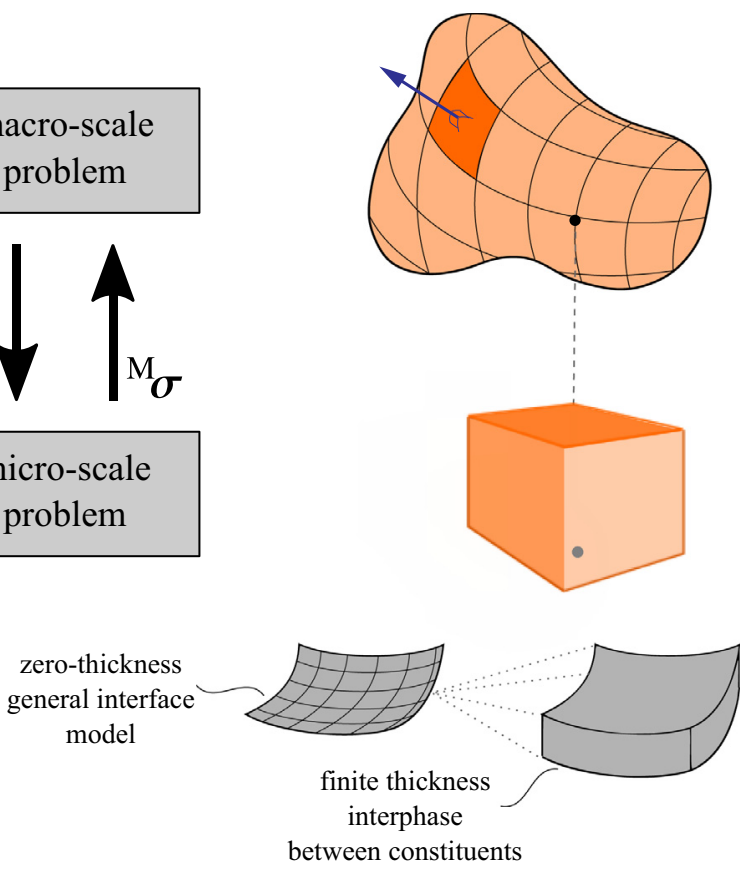

Fig. 3. A graphical summary of micro-to-macro transition accounting for general interfaces. The microscale corresponds to the representative volume element. Constitutive laws at the microscale are assumed to be known and the goal is to compute the effective response via homogenizing the response of the underlying microstructure. The zero-thickness interface model at the microscale replaces the finite-thickness interphase between different constituents.

model. Thus, the displacement of the interface itself $\overline{\boldsymbol{u}}$ remains unknown. A crucial, yet intuitively meaningful assumption, for the general interface model is that $\overline{\boldsymbol{u}}=\{\{\boldsymbol{u}\}\}$. That is, the interface coincides with the definition of the mid-surface across its minus and plus sides. The symmetric strains in the bulk and on the interface are defined by

$$
\boldsymbol{\varepsilon}=\frac{1}{2}\left[\boldsymbol{i} \cdot \operatorname{grad} \boldsymbol{u}+[\operatorname{grad} \boldsymbol{u}]^{\mathrm{t}} \cdot \boldsymbol{i}\right] \quad \text { in } \mathcal{B} \quad \text { and } \quad \overline{\boldsymbol{\varepsilon}}=\frac{1}{2}\left[\overline{\boldsymbol{i}} \cdot \overline{\operatorname{grad}} \overline{\boldsymbol{u}}+[\overline{\operatorname{grad}} \overline{\boldsymbol{u}}]^{\mathrm{t}} \cdot \overline{\boldsymbol{i}}\right] \quad \text { on } \mathcal{I},
$$

in which $\operatorname{grad}\{\bullet\}$ denotes the usual gradient operator. The interface gradient operator $\overline{\operatorname{grad}}\{\bullet\}$ is defined by the projection of the gradient operator onto the interface as $\overline{\operatorname{grad}}\{\bullet\}=\operatorname{grad}\{\bullet\} \cdot \overline{\boldsymbol{i}}$. Note, the definition of the bulk strain $(1)_{1}$ renders exactly the symmetric gradient of the displacement and its contraction with the identity tensor $\boldsymbol{i}$ does not alter its properties. That is not the case for the interface strain $(1)_{2}$ though. The contraction $\overline{\boldsymbol{i}} \cdot \overline{\operatorname{grad}} \overline{\boldsymbol{u}}$, where $\overline{\boldsymbol{i}}=\boldsymbol{i}-\overline{\boldsymbol{n}} \otimes \overline{\boldsymbol{n}}$, serves as a projection onto the interface. That is, the interface strain is not only the symmetric gradient of the interface displacement, but also its tangential component.

Governing equations of continua embedding the general interface model in small-strain elasticity theory can be obtained in a variationally consistent manner. To do so, the total energy is minimized via imposing the stationarity condition hence, setting its variations to zero. The total energy consists of the free energy in the bulk $\Psi$ and the free energy of the interface $\bar{\Psi}$ together with external contributions. The free energies in the bulk and on the interface are the integrals of their densities $\psi$ and $\bar{\psi}$, respectively, over their corresponding domains. The free energy density for the bulk is a function of the strain $\boldsymbol{\varepsilon}$ as $\psi=\psi(\varepsilon)$. Neglecting the scalar-valued liquid-like interface tension, the interface free energy density $\bar{\psi}$ depends on the interface strain $\overline{\boldsymbol{\varepsilon}}$ and the displacement jump $\llbracket \boldsymbol{u} \rrbracket$ as $\bar{\psi}=\bar{\psi}(\overline{\boldsymbol{\varepsilon}}, \llbracket \boldsymbol{u} \rrbracket)$. Constitutive relations in the bulk and on the interface connecting their corresponding energy conjugates are provided from the free energy densities as

$$
\boldsymbol{\sigma}=\frac{\partial \psi}{\partial \boldsymbol{\varepsilon}} \quad \text { in } \mathcal{B} \quad \text { and } \quad \overline{\boldsymbol{\sigma}}=\frac{\partial \bar{\psi}}{\partial \overline{\boldsymbol{\varepsilon}}}, \quad \overline{\boldsymbol{t}}=\frac{\partial \bar{\psi}}{\partial \llbracket \boldsymbol{u} \rrbracket} \quad \text { on } \mathcal{I},
$$

in which $\overline{\boldsymbol{t}}$ denotes the average traction across the interface or more precisely $\overline{\boldsymbol{t}}=\{\{\boldsymbol{\sigma}\} \cdot \overline{\boldsymbol{n}}$. Finally, the increments of the energy densities are

$$
\delta \psi=\boldsymbol{\sigma}: \delta \boldsymbol{\varepsilon} \quad \text { in } \mathcal{B} \quad \text { and } \quad \delta \bar{\psi}=\overline{\boldsymbol{\sigma}}: \delta \overline{\boldsymbol{\varepsilon}}+\overline{\boldsymbol{t}} \cdot \delta \llbracket \boldsymbol{u} \rrbracket
$$

Setting the variations of the total energy functional to zero for all admissible variations of displacements renders the governing equations. In the absence of external force densities within the bulk and on the interface, the governing equations 
for quasi-static problems read

$$
\begin{array}{ll}
\operatorname{div} \boldsymbol{\sigma}=\mathbf{0} & \text { in } \mathcal{B}, \\
\boldsymbol{\sigma} \cdot \widehat{\boldsymbol{n}}=\widehat{\boldsymbol{t}} & \text { on } \mathcal{S}, \\
\overline{\operatorname{div}} \overline{\boldsymbol{\sigma}}+\llbracket \boldsymbol{\sigma} \rrbracket \cdot \overline{\boldsymbol{n}}=\mathbf{0} & \text { on } \mathcal{I} \text { (along), } \\
\{\{\boldsymbol{\sigma}\} \cdot \overline{\boldsymbol{n}}=\overline{\boldsymbol{t}} & \text { on } \mathcal{I} \text { (across), } \\
\overline{\boldsymbol{\sigma}} \cdot \tilde{\boldsymbol{n}}=\tilde{\boldsymbol{t}} & \text { on } \mathcal{C},
\end{array}
$$

where $\widehat{\boldsymbol{t}}$ denotes the surface traction on $\mathcal{S}$ and similarly, $\tilde{\boldsymbol{t}}$ is the line traction on $\mathcal{C}$. The interface divergence operator is defined as $\overline{\operatorname{div}}\{\bullet\}=\operatorname{grad}\{\bullet\}: \overline{\boldsymbol{i}}$. It is of particular interest that the curvature of the interface is embedded within the interface divergence operator itself. Furthermore, the balance equation along the interface $(4)_{3}$ recovers the classical Young-Laplace equation, see Appendix A. For the sake of brevity, the details of derivations of the governing Eq. (4) are omitted here.

Next, the material behavior in the bulk and on the interface shall be discussed. The material response in the bulk is assumed to be standard and isotropic elastic according to the linear relation $\sigma=\mathbb{E}: \varepsilon$ in which $\mathbb{E}$ is the constitutive fourthorder tensor resulting in $\sigma=2 \mu \varepsilon+\lambda[\varepsilon: i] \boldsymbol{i}$ in which $\mu$ and $\lambda$ are the Lamé parameters. The behavior of the general interface model can be expressed using the constitutive laws

$$
\overline{\boldsymbol{\sigma}}=\overline{\mathbb{E}}: \overline{\boldsymbol{\varepsilon}}=2 \bar{\mu} \overline{\boldsymbol{\varepsilon}}+\bar{\lambda}[\overline{\boldsymbol{\varepsilon}}: \overline{\boldsymbol{i}}] \overline{\boldsymbol{i}} \quad, \quad \overline{\boldsymbol{t}}=\overline{\boldsymbol{k}} \cdot \llbracket \overline{\boldsymbol{u}} \rrbracket=\bar{k} \llbracket \overline{\boldsymbol{u}} \rrbracket .
$$

The fourth-order constitutive tensor $\overline{\mathbb{E}}$ and the symmetric second-order positive definite tensor $\overline{\boldsymbol{k}}$ correspond to the interface response along and across the interface, respectively. Here, $\bar{\mu}$ and $\bar{\lambda}$ denote the interface Lamé parameters and $\bar{k}$ is the cohesive resistance of the interface. Finally, it is straightforward to formulate the bulk and interface energies, respectively as

$$
\begin{aligned}
\delta \psi=\boldsymbol{\sigma}: \delta \boldsymbol{\varepsilon} & \Rightarrow & \psi=\frac{1}{2} \boldsymbol{\varepsilon}: \mathbb{E}: \boldsymbol{\varepsilon} \\
\delta \bar{\psi}=\overline{\boldsymbol{\sigma}}: \delta \overline{\boldsymbol{\varepsilon}}+\overline{\boldsymbol{t}} \cdot \delta \llbracket \overline{\boldsymbol{u}} \rrbracket & \Rightarrow & \psi=\frac{1}{2} \overline{\boldsymbol{\varepsilon}}: \overline{\mathbb{E}}: \overline{\boldsymbol{\varepsilon}}+\frac{1}{2} \overline{\boldsymbol{k}}: \llbracket \overline{\boldsymbol{u}} \rrbracket \otimes \llbracket \overline{\boldsymbol{u}} \rrbracket .
\end{aligned}
$$

Obviously, the constitutive model (5) corresponds to an isotropic interface behavior on the interface tangent plane. The nature of the Lamé parameters is similar to the two-dimensional plane stress elasticity and they both correlate with the resistance of the interface against deformations tangent to its plane. On the other hand, the interface cohesive parameter $\bar{k}$ describes the resistance of the interface against opening and can be understood as orthogonal resistance of the interface. In particular case of fiber composites here, the interface parameter $\bar{\lambda}$ can be set to zero since only one parameter suffices to define the interface behavior. In this case, the interface parameter $\bar{\mu}$ describes the resistance of the interface against the change of its length.

In passing, we mention that the current interface model neglects the contributions from the interface tension along the interface. More precisely, we study solely the size dependent elastic response of the material. Including the interface tension in the current framework does not add additional insight to the final results nor it requires further effort in the framework while it introduces a more complex notation. Furthermore, accounting for the interface tension requires dealing with effective interface elastic constants instead of the Lamé parameters. The parameter identification in Miller et al. (2000) reveals that the influence of the interface tension in solids is generally expected to be considerably smaller than that of the interface elastic resistance. The interface tension essentially results in a residual stress in the material that resembles the pore pressure in the homogenization of porous media. Obviously, the most general framework for the interface elasticity theory must include the interface tension as well as the interface elasticity, see Huang and Wang (2006); 2013), Javili et al. among others.

\subsection{Micro to macro transition}

The goal of this section is to provide an energetically consistent micro-to-macro transition framework. That is, to elabo-

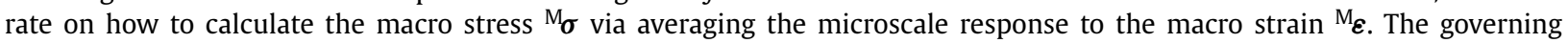
equations at the macroscale are standard and can be summarized as



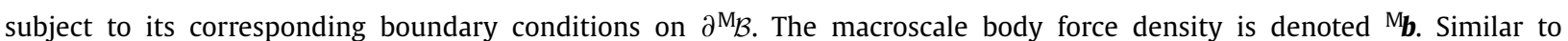
the microscale, the macroscale energy density ${ }^{\mathrm{M}} \psi$ can be assigned to the body. The incremental energy density at the macroscale can be identified as

$$
\delta^{\mathrm{M}} \psi={ }^{\mathrm{M}} \boldsymbol{\sigma}: \delta^{\mathrm{M}} \boldsymbol{\varepsilon}
$$

A commonly accepted strategy to connect the macroscale and microscale measures is the volume averaging of the microscale quantities over the RVE. Due to the presence of the non-standard general interface model at the microscale, such 
Table 1

Summary of the micro-to-macro transition accounting for general interfaces. The macroscopic quantities are expressed as integrals at the microscale. In the absence of the jump across the interface and the elastic response along the interface, the integrals reduce to their familiar formats in the classical homogenization.

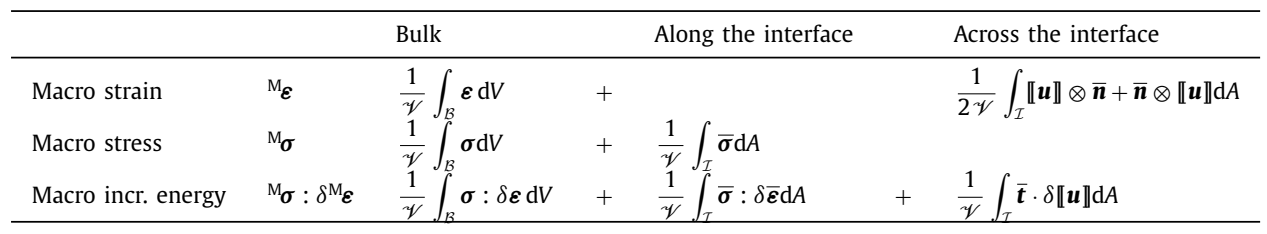

classical definitions must be carefully examined and extended appropriately. Let $\mathscr{V}$ denotes the total volume of the RVE which can be computed by

$$
\mathscr{V} \boldsymbol{i}=\int_{\mathcal{S}} \boldsymbol{x} \otimes \boldsymbol{n} \mathrm{d} A \quad \text { or } \quad \mathscr{V}=\int_{\mathcal{B}} \mathrm{d} V=\frac{1}{3} \int_{\mathcal{S}} \boldsymbol{x} \cdot \boldsymbol{n} \mathrm{d} A .
$$

The macro strain as the average of the surface integral over the boundary of the RVE reads

$$
{ }^{\mathrm{M}} \boldsymbol{\varepsilon}=\frac{1}{\mathscr{V}} \int_{\mathcal{S}} \frac{1}{2}[\boldsymbol{u} \otimes \boldsymbol{n}+\boldsymbol{n} \otimes \boldsymbol{u}] \mathrm{d} A .
$$

Through appropriate divergence theorems in the bulk and on the interface, the surface integral (10) can be transformed into a combination of integrals over the $\operatorname{RVE} \mathcal{B}$ and on the interface $\mathcal{I}$ as

$$
\mathrm{M}_{\boldsymbol{\varepsilon}}=\frac{1}{\mathscr{V}} \int_{\mathcal{B}} \boldsymbol{\varepsilon} \mathrm{d} V+\frac{1}{\mathscr{V}} \int_{\mathcal{I}} \frac{1}{2}[\llbracket \boldsymbol{u} \rrbracket \otimes \overline{\boldsymbol{n}}+\overline{\boldsymbol{n}} \otimes \llbracket \boldsymbol{u} \rrbracket] \mathrm{d} A .
$$

Clearly, the second term corresponds to the displacement jump across the interface and vanishes in the absence of interfaces resulting in the classical definition of the macro strain.

Next, the macro stress ${ }^{\mathrm{M}} \boldsymbol{\sigma}$ should be linked to the microscale behavior. Motivated by the average stress theorem, the macro stress can be defined by integrals over the boundary of the RVE as

$$
\mathrm{M}^{\mathrm{\sigma}}=\frac{1}{\mathscr{V}} \int_{\mathcal{S}} \widehat{\boldsymbol{t}} \otimes \widehat{\boldsymbol{x}} \mathrm{d} A+\frac{1}{\mathscr{V}} \int_{\mathcal{C}} \tilde{\boldsymbol{t}} \otimes \tilde{\boldsymbol{x}} \mathrm{d} L
$$

Obviously, the integral over the curve $\mathcal{C}$ vanishes for closed interfaces entirely embedded within the RVE. Similar to the macro strain, the macro stress ${ }^{{ }^{M}} \boldsymbol{\sigma}$ can be expressed (e.g. Chatzigeorgiou et al., 2015) in terms of integrals over $\mathcal{B}$ and $\mathcal{I}$ as

$$
{ }^{\mathrm{M}} \boldsymbol{\sigma}=\frac{1}{\mathscr{V}} \int_{\mathcal{B}} \boldsymbol{\sigma} \mathrm{d} V+\frac{1}{\mathscr{V}} \int_{\mathcal{I}} \bar{\sigma} \mathrm{d} A
$$

The second integral corresponds to the interface stress and vanishes in the absence of elasticity along the interface. Note, the interface average traction $\overline{\boldsymbol{t}}$ does not explicitly enters the definition of the macro stress. Table 1 summarizes the microto-macro transition of continua accounting for general interfaces.

Equipped with the definitions of the macro strain ${ }^{{ }^{*}} \boldsymbol{\varepsilon}$ and macro stress ${ }^{{ }^{N}} \boldsymbol{\sigma}$ in terms of microscale quantities, the last step of a physically meaningful homogenization is to guarantee the incremental energy equivalence between the two scales. The incremental energy equivalence between the scales is often referred to as the macro homogeneity condition or the Hill-Mandel condition and can be expressed as

$$
\delta^{\mathrm{M}} \psi \stackrel{!}{=} \frac{1}{\mathscr{V}} \int_{\mathcal{B}} \delta \psi \mathrm{d} V+\frac{1}{\mathscr{V}} \int_{\mathcal{I}} \delta \bar{\psi} \mathrm{d} A
$$

in which the sign $\stackrel{!}{=}$ denotes the conditional equality. Inserting the incremental micro and macro energies (3) and (8), respectively into the Hill-Mandel condition (14) and collecting all the terms on one side yields

$$
\frac{1}{\mathscr{V}} \int_{\mathcal{B}} \boldsymbol{\sigma}: \delta \boldsymbol{\varepsilon} \mathrm{d} V+\frac{1}{\mathscr{V}} \int_{\mathcal{I}} \overline{\boldsymbol{\sigma}}: \delta \overline{\boldsymbol{\varepsilon}}+\overline{\boldsymbol{t}} \cdot \delta \llbracket \boldsymbol{u} \rrbracket \mathrm{d} A-{ }^{\mathrm{M}} \boldsymbol{\sigma}: \delta{ }^{\mathrm{M}} \boldsymbol{\varepsilon} \stackrel{!}{=} 0
$$

Finally, replacing the macro strain (10) and macro stress (12) into the left-hand side of the expanded Hill-Mandel condition (15) after some mathematical steps results in the identity

$$
\frac{1}{\mathscr{V}} \int_{\mathcal{B}} \boldsymbol{\sigma}: \delta \boldsymbol{\varepsilon} \mathrm{d} V+\frac{1}{\mathscr{V}} \int_{\mathcal{I}} \overline{\boldsymbol{\sigma}}: \delta \overline{\boldsymbol{\varepsilon}}+\overline{\boldsymbol{t}} \cdot \delta \llbracket \boldsymbol{u} \rrbracket \mathrm{d} A-{ }^{\mathrm{M}} \boldsymbol{\sigma}: \delta^{\mathrm{M}} \boldsymbol{\varepsilon}=\frac{1}{\mathscr{V}} \int_{\mathcal{S}}\left[\delta \boldsymbol{u}-\delta^{\mathrm{M}} \boldsymbol{\varepsilon} \cdot \widehat{\boldsymbol{x}}\right] \cdot\left[\widehat{\boldsymbol{t}}-{ }^{\mathrm{M}} \boldsymbol{\sigma} \cdot \widehat{\boldsymbol{n}}\right] \mathrm{d} A,
$$

for closed interfaces. The identity (16) is essentially an extended Hill's lemma accounting for the general interface model. Obviously, in the absence of interfaces at the microscale this identity reduces to the classical Hill's lemma. Utilizing the identity (16) in the expanded Hill-Mandel condition (15), renders an alternative format of the Hill-Mandel condition as

$$
\int_{\mathcal{S}}\left[\delta \boldsymbol{u}-\delta^{\mathrm{M}} \boldsymbol{\varepsilon} \cdot \widehat{\boldsymbol{x}}\right] \cdot\left[\widehat{\boldsymbol{t}}-{ }^{\mathrm{M}} \boldsymbol{\sigma} \cdot \widehat{\boldsymbol{n}}\right] \mathrm{d} A \stackrel{!}{=} 0
$$



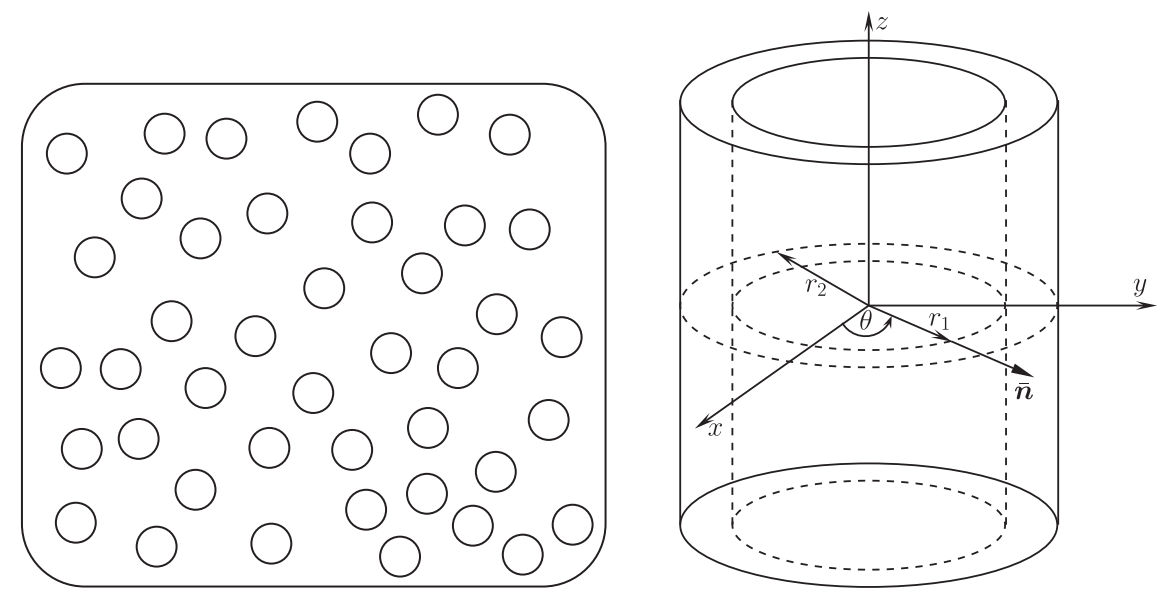

Fig. 4. Unidirectional fiber composite and its RVE, according to the composite cylinders method.

The significance of the condition (17) is that it furnishes suitable boundary conditions for the microscale problem. All boundary conditions that satisfy the condition (17) sufficiently fulfill the Hill-Mandel condition (14) and thus, are admissible choices for an energetically consistent homogenization. Three canonical boundary conditions are (1) linear displacements (2) constant traction and (3) periodic displacement and anti-periodic traction.

\section{Effective properties of unidirectional fiber composites}

This section elaborates on an analytical approach to obtain the effective properties of a unidirectional fiber composite embedding generalized interfaces. The problem of composites with interfaces has been studied extensively in the literature of micromechanics methods, see for instance Benveniste and Miloh (2001); Hashin (1990) among others. In several approaches the interface is captured by considering a very thin interphase (Benveniste, 2013a; Hashin, 2002). For zerothickness cohesive interfaces, Hashin has performed numerous studies (Hashin, 1990; 1991; 1992). In the case of elastic interfaces, studies based on particulate or unidirectional fiber composites exist in the literature (Duan et al., 2005b; 2006; Sharma and Ganti, 2004). The main issue with the elastic interfaces is that the mean field theories can only be applied for reinforcements of constant curvature (spherical particles or cylindrical fibers), due to the fact that in the other cases the Eshelby problem does not provide uniform elastic state inside the inclusion as pointed out by Sharma and Ganti (2004). Duan et al. (2005a) have shown that the elastic interfaces cause the Eshelby tensor and the stress concentration tensors to be non-uniform and size dependent. This restriction of course is also extended in the case of generalized interfaces and thus, numerical approaches are unavoidable for short fiber composites or composites with reinforcement of ellipsoidal form. Gu et al. (2014) have provided analytical forms for the elastic moduli of spherical particulate composites with generalized interfaces. Chen et al. (2016) have studied the effect of residual interface stress on thermo-elastic properties of unidirectional fiber composites.

For a unidirectional fiber composite, the RVE can be well defined and consists of a cylindrical fiber surrounded by the matrix material. As Hashin and Rosen (1964) have illustrated, the RVE can be described as a system of concentric cylinders as shown in Fig. 4. The radius of the fiber is denoted $r_{1}$, while the external radius of the matrix surrounding the fiber is denoted as $r_{2}$. The form of the RVE makes preferable to write all the necessary equations of the problem in cylindrical coordinates. In this coordinate system the main axes are the radius $r$, the angle $\theta$ and the axis parallel to the fiber axis $z$. When both the fiber (phase 1) and the matrix (phase 2) are linear isotropic materials with Lame constants $\lambda$ and $\mu$, the stresses and the strains in both phases are connected through the equations

$$
\begin{array}{lll}
\sigma_{r r}^{(i)}=\left[\lambda^{(i)}+2 \mu^{(i)}\right] \varepsilon_{r r}^{(i)}+\lambda^{(i)} \varepsilon_{\theta \theta}^{(i)}+\lambda^{(i)} \varepsilon_{z z}^{(i)}, & & \sigma_{\theta \theta}^{(i)}=\lambda^{(i)} \varepsilon_{r r}^{(i)}+\left[\lambda^{(i)}+2 \mu^{(i)}\right] \varepsilon_{\theta \theta}^{(i)}+\lambda^{(i)} \varepsilon_{z z}^{(i)}, \\
\sigma_{z z}^{(i)}=\lambda^{(i)} \varepsilon_{r r}^{(i)}+\lambda^{(i)} \varepsilon_{\theta \theta}^{(i)}+\left[\lambda^{(i)}+2 \mu^{(i)}\right] \varepsilon_{z z}^{(i)}, & & \sigma_{r z}^{(i)}=\sigma_{z r}=2 \mu^{(i)} \varepsilon_{r z}^{(i)}, \\
\sigma_{\theta z}^{(i)}=\sigma_{z \theta}=2 \mu^{(i)} \varepsilon_{z \theta}^{(i)}, & & \sigma_{r \theta}^{(i)}=\sigma_{\theta r}=2 \mu^{(i)} \varepsilon_{r \theta}^{(i)} .
\end{array}
$$

while the kinematic conditions provide the relations between the strains and displacements

$$
\begin{array}{lll}
\varepsilon_{r r}^{(i)}=\frac{\partial u_{r}^{(i)}}{\partial r}, & \varepsilon_{\theta \theta}^{(i)}=\frac{1}{r} \frac{\partial u_{\theta}^{(i)}}{\partial \theta}+\frac{u_{r}^{(i)}}{r}, & \varepsilon_{z z}^{(i)}=\frac{\partial u_{z}^{(i)}}{\partial z}, \\
2 \varepsilon_{r z}=\frac{\partial u_{z}^{(i)}}{\partial r}+\frac{\partial u_{r}^{(i)}}{\partial z}, & 2 \varepsilon_{\theta z}=\frac{1}{r} \frac{\partial u_{z}^{(i)}}{\partial \theta}+\frac{\partial u_{\theta}^{(i)}}{\partial z}, & 2 \varepsilon_{r \theta}=\frac{\partial u_{\theta}^{(i)}}{\partial r}+\frac{1}{r} \frac{\partial u_{r}^{(i)}}{\partial \theta}-\frac{u_{\theta}^{(i)}}{r} .
\end{array}
$$

The index $i$ takes the value 1 for the fiber and 2 for the matrix. For the general interface, as discussed in the previous section, the displacement is equal to the average of the displacements at the borders of the two material phases as $\overline{\boldsymbol{u}}=\{\{\boldsymbol{u}\}$. 


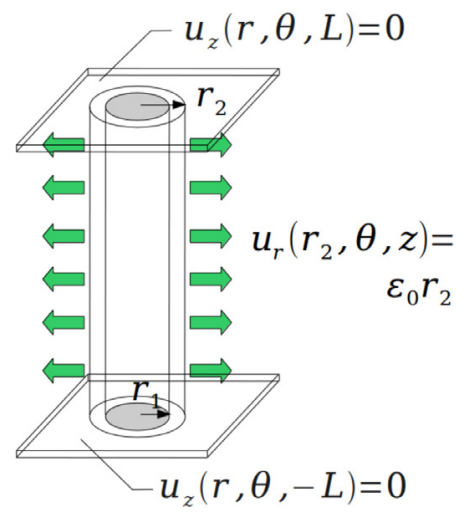

Fig. 5. Fiber composite RVE under radial strain conditions.

On the other hand, for isotropic interface with zero surface Lame constant $\bar{\lambda}$ the interface stresses and strains are connected through the relations

$$
\bar{\sigma}_{\theta \theta}=2 \bar{\mu} \bar{\varepsilon}_{\theta \theta}, \quad \bar{\sigma}_{z z}=2 \bar{\mu} \bar{\varepsilon}_{z z}, \quad \bar{\sigma}_{\theta z}=2 \bar{\mu} \bar{\varepsilon}_{\theta z},
$$

where the interface strains are expressed in terms of the interface displacements as

$$
\bar{\varepsilon}_{\theta \theta}=\frac{1}{r_{1}} \frac{\partial \bar{u}_{\theta}}{\partial \theta}+\frac{\bar{u}_{r}}{r_{1}}, \quad \quad \bar{\varepsilon}_{z z}=\frac{\partial \bar{u}_{z}}{\partial z}, \quad 2 \bar{\varepsilon}_{\theta z}=\frac{1}{r_{1}} \frac{\partial \bar{u}_{z}}{\partial \theta}+\frac{\partial \bar{u}_{\theta}}{\partial z} .
$$

The interface equilibrium Eq. $(4)_{3}$ holds on the interface at $r=r_{1}$ and can be expressed in cylindrical coordinates as

$$
[\overline{\operatorname{div}} \overline{\boldsymbol{\sigma}}]_{r}+\llbracket t_{r} \rrbracket=0, \quad[\overline{\operatorname{div}} \overline{\boldsymbol{\sigma}}]_{\theta}+\llbracket t_{\theta} \rrbracket=0, \quad[\overline{\operatorname{div}} \overline{\boldsymbol{\sigma}}]_{z}+\llbracket t_{z} \rrbracket=0,
$$

or alternatively

$$
-\frac{\bar{\sigma}_{\theta \theta}}{r_{1}}+\llbracket \sigma_{r r} \rrbracket=0, \quad \frac{1}{r_{1}} \frac{\partial \bar{\sigma}_{\theta \theta}}{\partial \theta}+\frac{\partial \bar{\sigma}_{\theta z}}{\partial z}+\llbracket \sigma_{r \theta} \rrbracket=0, \quad \frac{1}{r_{1}} \frac{\partial \bar{\sigma}_{\theta z}}{\partial \theta}+\frac{\partial \bar{\sigma}_{z z}}{\partial z}+\llbracket \sigma_{r z} \rrbracket=0 .
$$

The average traction on the interface $(4)_{4}$ satisfies the general form $\{\boldsymbol{\sigma} \boldsymbol{\sigma}\} \cdot \overline{\boldsymbol{n}}=\bar{k} \llbracket \boldsymbol{u} \rrbracket$. Equipped with these relations, in the next subsections two special cases are examined. First, a dilatational expansion of the RVE in the direction normal to the fibers axis second, shearing of the RVE in the direction normal to the fibers axis.

\subsection{Dilatational expansion - in plane bulk modulus}

Consider that the RVE of the fiber composite is subjected to constant radial strain ${ }^{\mathrm{M}_{\varepsilon_{r r}}}=\varepsilon_{0}$ and it is fixed on upper and lower surfaces (Fig. 5). This is a well defined problem for elastic materials and analytical solutions are provided in the literature when the interfaces are perfect, i.e. no jumps in tractions and displacements (Hashin and Rosen, 1964). Analytical solutions also exist for pure elasticity in the cases where either jump in displacements is allowed (Hashin, 1991) or jump in tractions is allowed (Duan et al., 2006).

Hashin and Rosen (1964) have shown that, under these boundary conditions, the displacement is zero at the $\theta$ and $z$ directions, while the displacement fields in the $r$ direction are expressed as

$$
u_{r}^{(i)}=D_{1}^{(i)} r+D_{2}^{(i)} \frac{1}{r}, \quad i=1,2 .
$$

For these displacement fields, the strains and stresses for the fiber, the matrix and the general interface are expressed with the help of the relations (18)-(21). The elasticity problem in that case is reduced on calculating $D_{1}^{(1)}, D_{2}^{(1)}, D_{1}^{(2)}$ and $D_{2}^{(2)}$. These constants are obtained with the help of the overall boundary and compatibility conditions, as well as the jump conditions at the interface:

- Finite solution at the center of the fiber

$$
u_{r} \text { finite at } r=0 \text { or } \quad D_{2}^{(1)}=0 .
$$

- Boundary condition at the outer cylinder

$$
u_{r}^{(2)}\left(r_{2}\right)=\varepsilon_{0} r_{2} .
$$

- Traction equilibrium at the interface

$$
-\frac{\bar{\sigma}_{\theta \theta}}{r_{1}}+\sigma_{r r}^{(2)}\left(r_{1}\right)-\sigma_{r r}^{(1)}\left(r_{1}\right)=0 .
$$



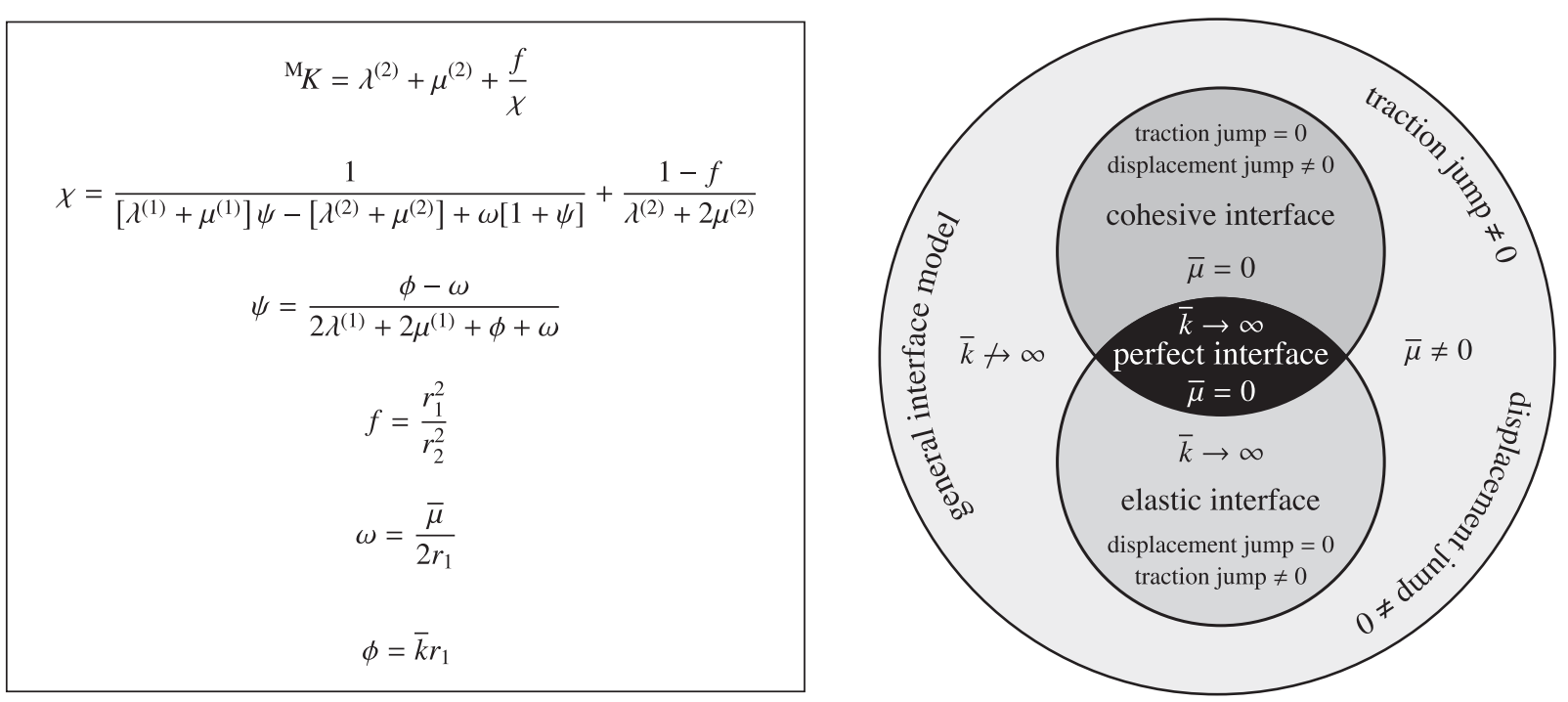

Fig. 6. An overview of interface models with respect to the effective bulk modulus. Explicit expression for the effective bulk modulus ${ }_{K} K$ (left) and its degeneration to existing models (right). The superscript index take the value 1 for the fiber and 2 for the matrix.

- The average traction at the interface

$$
\frac{\sigma_{r r}^{(2)}\left(r_{1}\right)+\sigma_{r r}^{(1)}\left(r_{1}\right)}{2}=\bar{k}\left[u_{r}^{(2)}\left(r_{1}\right)-u_{r}^{(1)}\left(r_{1}\right)\right] .
$$

The system of Eqs. (24)-(27) is linear and can be solved explicitly. Then, all the stresses, strains and displacements are obtained analytically. Due to the uniformity of the traction vector at the boundary, the macroscopic radial stress is given by ${ }^{\mathrm{M}} \sigma_{r r}=\sigma_{r r}^{(2)}\left(r_{2}\right)$ according to Hashin and Rosen (1964). Thus, the in plane bulk modulus ${ }^{\mathrm{M}_{K}}$ of the fiber composite is obtained (Christensen, 1979) as

$$
\mathrm{M}_{K}=\frac{{ }^{\mathrm{M}} \sigma_{r r}}{2 \mathrm{M}_{\varepsilon_{r r}}}=\frac{\sigma_{r r}^{(2)}\left(r_{2}\right)}{2 \varepsilon_{0}} .
$$

For a unidirectional fiber composite presenting general interfaces between the matrix and the fibers, it is possible to provide an explicit expression for the effective in-plane bulk modulus as

$$
\mathrm{M}_{K}=\lambda^{(2)}+\mu^{(2)}+\frac{f}{\chi} \quad \text { with } \quad \chi=\frac{1}{\left[\lambda^{(1)}+\mu^{(1)}\right] \psi-\left[\lambda^{(2)}+\mu^{(2)}\right]+\omega[1+\psi]}+\frac{1-f}{\lambda^{(2)}+2 \mu^{(2)}}, \quad f=\frac{r_{1}^{2}}{r_{2}^{2}},
$$

in which

$$
\omega=\frac{\bar{\mu}}{2 r_{1}}, \quad \phi=\bar{k} r_{1}, \quad \psi=\frac{\phi-\omega}{2 \lambda^{(1)}+2 \mu^{(1)}+\phi+\omega},
$$

and the fiber to matrix volume fraction is denote $f$ although in two dimensions.

The explicit expression for the effective bulk modulus (29), which is also summarized in Fig. 6, is particularly interesting since it reveals the connection of the current contribution to the existing ones. In fact, it is relatively straightforward to show how the effective bulk modulus recovers the classical cases in its simplified forms as follows.

- Perfect interface model can be recovered by setting $\phi \rightarrow \infty$ since the orthogonal resistance of the interface against opening $\bar{k} \rightarrow \infty$ and the interface remain geometrically coherent regardless of the deformation. For the perfect interface model, the elastic resistance along the interface vanishes and thus $\bar{\mu}=0$ which results in $\omega=0$. Eventually, since $\phi \rightarrow \infty$ and the material parameters of the fiber are finite, then $\psi=1$. Inserting all these into Eq. (29) furnishes the effective inplane bulk modulus for the perfect interface model which is precisely the same result obtained in the seminal work of Hashin and Rosen (1964).

- Elastic interface model is geometrically coherent and does not allow for opening and hence, the cohesive resistance of the interface tends $\bar{k}$ to infinity resulting in $\phi \rightarrow \infty$ and consequently $\psi=1$. However, unlike the perfect interface model, the elastic resistance along the interface $\bar{\mu}$ does not vanish and assumes a finite value. The final expression for the elastic interface model shall be compared with the findings in Duan et al. (2009); 2006); 2007) among others. 


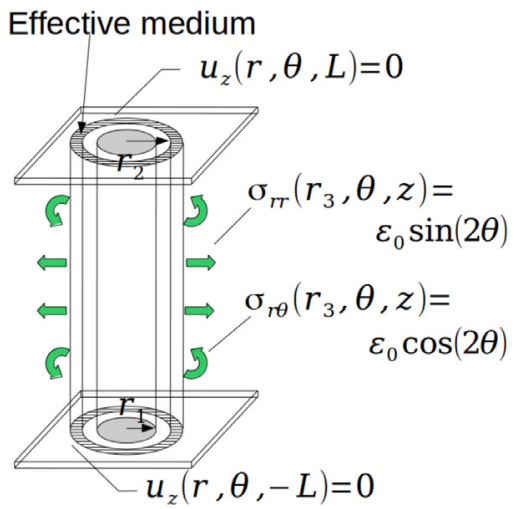

Fig. 7. Fiber composite RVE under shear conditions normal to the fiber axis.

- Cohesive interface model does not possess an elastic resistance along the interface. That is, for the cohesive interface model $\bar{\mu}=0$ resulting in $\omega=0$. The parameter $\phi$ however remains finite and plays a crucial role since it is proportional to the cohesive resistance $\bar{k}$ defining the orthogonal behavior of the interface. The parameter $\psi$ reads $\psi=\phi /\left[2 \lambda^{(1)}+2 \mu^{(1)}+\phi\right]$. Inserting all these into Eq. (29) furnishes the effective in-plane bulk modulus for the cohesive interface model which is precisely the same result obtained in Hashin (1990).

- General interface model allows for a finite resistance along the interface $\bar{\mu}$ as well as a finite resistance orthogonal to the interface $\bar{k}$. In this case, both parameters $\omega$ and $\phi$ assume finite values resulting in a finite $\psi$ not necessarily being one nor simplified.

\subsection{Transverse shearing - in plane shear modulus}

To identify the in plane shear modulus of a unidirectional composite, Hashin and Rosen (1964) have proposed the boundary value problem illustrated in Fig. 7, which correspond to simple shear stress in the normal plane to the fibers. They compared the solution obtained when the external stress conditions are substituted by boundary displacements of the same type. The obtained in plane shear moduli from these two problems present a small difference, thus the original form of the problem provides only bounds on this modulus. To resolve this issue, Christensen and Lo (1979) have developed a modification of the original problem, by adding an external third layer, whose properties are those of the unknown composite medium. This way, the composite cylinders method is transformed to the generalized self consistent composite cylinders method.

In the modified version in Christensen and Lo (1979), the boundary value problem of Fig. 7 has $u_{z}$ equal to zero everywhere and the rest of displacement fields are given by

$$
\begin{aligned}
& u_{r}^{(i)}=\sum_{j=1}^{4} a_{j}^{(i)} D_{j}^{(i)} r^{(i)} \sin (2 \theta), \quad u_{\theta}^{(i)}=\sum_{j=1}^{4} D_{j}^{(i)} r^{n_{j}^{(i)}} \cos (2 \theta), \quad i=1,2, \\
& u_{r}^{(3)}=\varepsilon_{0} \frac{r_{2}}{4 \mathrm{M} \mu}\left[\frac{2 r}{r_{2}}+D_{3}^{(3)} \frac{r_{2}^{3}}{r^{3}}+2\left[1+\frac{\mathrm{M}_{\mu}}{\mathrm{M} K}\right] D_{4}^{(3)} \frac{r_{2}}{r}\right] \sin (2 \theta), \\
& u_{\theta}^{(3)}=\varepsilon_{0} \frac{r_{2}}{4^{\mathrm{M}} \mu}\left[\frac{2 r}{r_{2}}-D_{3}^{(3)} \frac{r_{2}^{3}}{r^{3}}+2 \frac{\mathrm{M}_{\mu}}{\mathrm{M} K} D_{4}^{(3)} \frac{r_{2}}{r}\right] \cos (2 \theta),
\end{aligned}
$$

where

$$
a_{j}^{(i)}=\frac{2 \lambda^{(i)}+6 \mu^{(i)}-2 n_{j}^{(i)}\left[\lambda^{(i)}+\mu^{(i)}\right]}{\lambda^{(i)}+6 \mu^{(i)}-\left[n_{j}^{(i)}\right]^{2}\left[\lambda^{(i)}+2 \mu^{(i)}\right]},
$$

and $n_{j}^{(i)}$ are the solutions of the polynomial $n^{4}-10 n^{2}+9=0$. This equation leads to four roots, namely $n_{j}^{(1)}=3, n_{j}^{(2)}=1$, $n_{j}^{(3)}=-3, n_{j}^{(4)}=-1$. Moreover, $\mathrm{M}_{\mu}$ is the unknown in plane shear modulus of the fiber composite. For these displacement fields, the strains and stresses for all the phases (fiber, matrix, effective medium) and the general interface are expressed in terms of the unknown $D_{j}^{(i)}$ constants with the help of the relations (18)-(21). The boundary and interface conditions that hold in the RVE in this problem are the following:

- Finite solution at the center of the fiber

$$
u_{r}, u_{\theta} \text { finite at } r=0 \text { or } D_{3}^{(1)}=D_{4}^{(1)}=0 \text {. }
$$




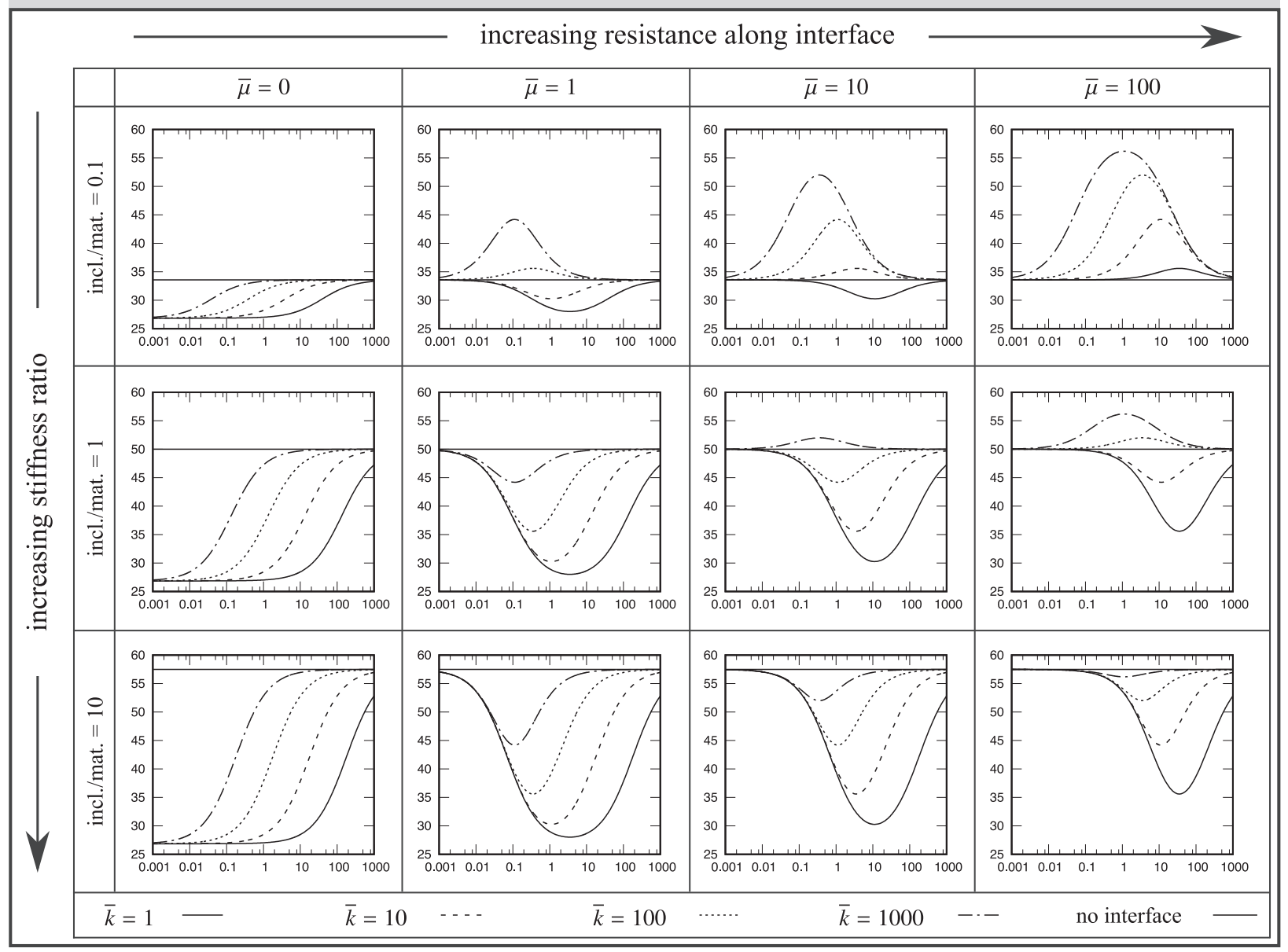

Fig. 8. Effective bulk modulus versus size of the RVE for the volume fraction of $12.57 \%$. Increasing the resistance against the change of length along the interface correlates to increasing the interface parameter $\bar{\mu}$. Different lines on each graph correspond to different orthogonal resistance of the interface against opening, see the legend at the bottom. In the absence of the interface, the size effect is completely ruled out resulting in a constant effective response.

- Traction continuity at the interface between effective medium and matrix

$$
\sigma_{r r}^{(2)}\left(r_{2}\right)=\sigma_{r r}^{(3)}\left(r_{2}\right), \quad \sigma_{r \theta}^{(2)}\left(r_{2}\right)=\sigma_{r \theta}^{(3)}\left(r_{2}\right) .
$$

- Displacement continuity at the interface between effective medium and matrix

$$
u_{r}^{(2)}\left(r_{2}\right)=u_{r}^{(3)}\left(r_{2}\right), \quad u_{\theta}^{(2)}\left(r_{2}\right)=u_{\theta}^{(3)}\left(r_{2}\right) .
$$

- Traction equilibrium equations at the interface between fiber and matrix

$$
-\frac{\bar{\sigma}_{\theta \theta}}{r_{1}}+\sigma_{r r}^{(2)}\left(r_{1}\right)-\sigma_{r r}^{(1)}\left(r_{1}\right)=0, \quad \frac{1}{r_{1}} \frac{\partial \bar{\sigma}_{\theta \theta}}{\partial \theta}+\sigma_{r \theta}^{(2)}\left(r_{1}\right)-\sigma_{r \theta}^{(1)}\left(r_{1}\right)=0 .
$$

- Traction averages at the interface between fiber and matrix

$$
\frac{\sigma_{r r}^{(2)}\left(r_{1}\right)+\sigma_{r r}^{(1)}\left(r_{1}\right)}{2}=\bar{k}\left[u_{r}^{(2)}\left(r_{1}\right)-u_{r}^{(1)}\left(r_{1}\right)\right], \quad \frac{\sigma_{r \theta}^{(2)}\left(r_{1}\right)+\sigma_{r \theta}^{(1)}\left(r_{1}\right)}{2}=\bar{k}\left[u_{\theta}^{(2)}\left(r_{1}\right)-u_{\theta}^{(1)}\left(r_{1}\right)\right] .
$$

The above ten equations are not sufficient to identify the ten unknown constants and the unknown in plane shear modulus. The last necessary condition arise from the energetic criterion identified in Christensen and Lo (1979), which provides the simple result $D_{4}^{(3)}=0$. After proper calculations, the unknown ${ }^{\mathrm{M}} \mu$ is identified with the help of a quadratic equation, which has one positive and one negative solution. The latter of course is ignored. Unlike the effective bulk modulus ${ }^{M_{K}}$, it is impossible to provide a simple explicits expression for the effective shear modulus ${ }^{\mathrm{M}} \mu$ and identify its simplified forms 


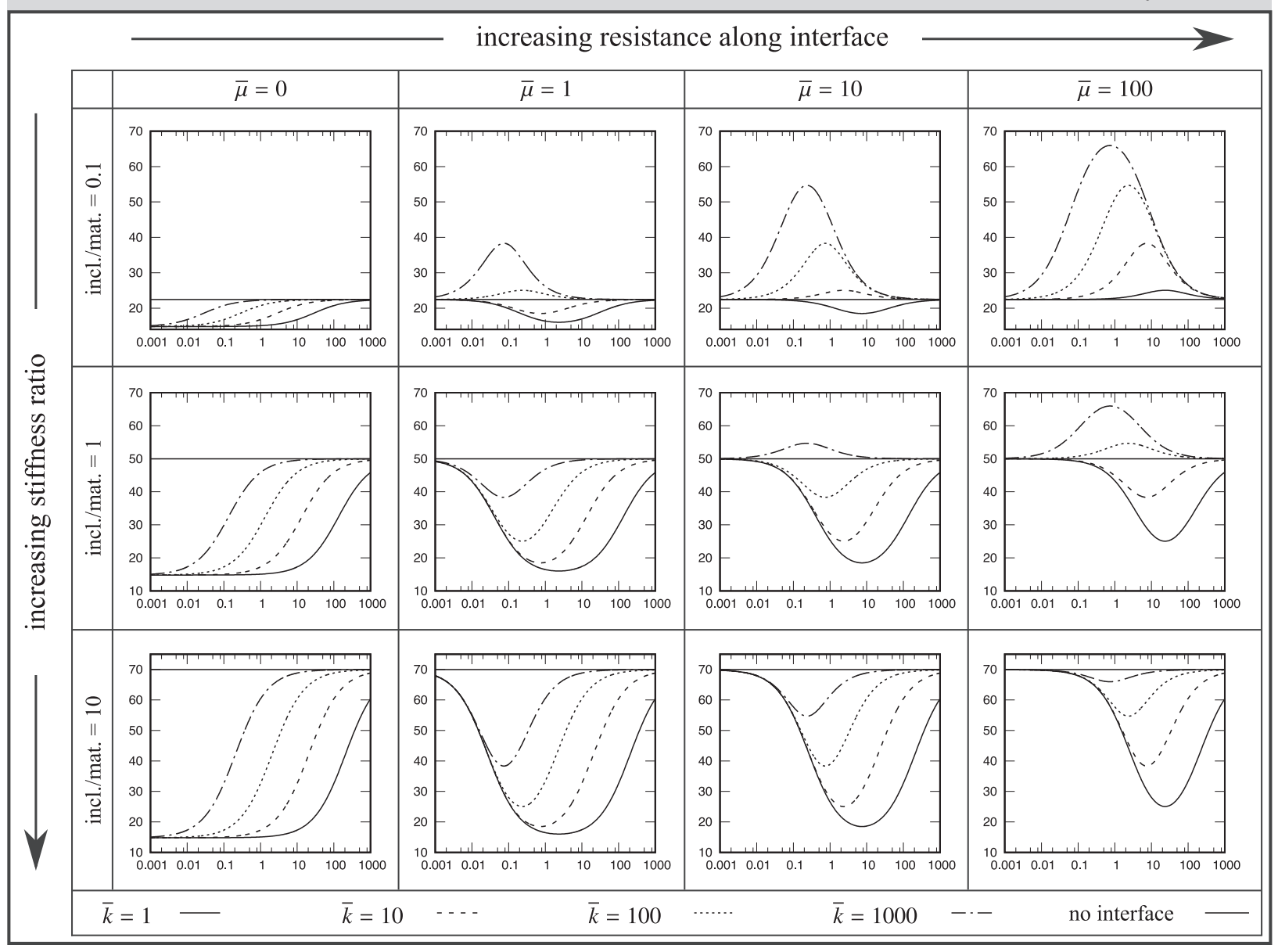

Fig. 9. Effective bulk modulus versus size of the RVE for the volume fraction of $28.27 \%$. Increasing the resistance against the change of length along the interface correlates to increasing the interface parameter $\bar{\mu}$. Different lines on each graph correspond to different orthogonal resistance of the interface against opening, see the legend at the bottom. In the absence of the interface, the size effect is completely ruled out resulting in a constant effective response.

for various interface types. Nonetheless, for the sake of completeness, Appendix B provides a semi-explicit form for the effective shear modulus and its degeneration to recover different interface models. Further effective elastic constants of fiber composites are elaborated in Appendix C.

\section{Examples}

The goal of this section is to elucidate the theory via a series of numerical examples. In particular, the influence of generalized interfaces on the overall response of a material is investigated. The forthcoming examples include a large variety of parametric studies to cover various scenarios. The numerical simulations are realized over a square RVE with the fiber exactly at its center. All studies are carried out for three volume fractions of $f=12.57 \%, f=28.27 \%$ and $f=50.26 \%$. For a tetragonal packing with a square unit cell of the length $\ell$, the three volume fractions correspond to $r / \ell=0.2, r / \ell=0.3$ and $r / \ell=0.4$, respectively, where $r$ is the radius of the fiber. The length of the unit cell $\ell$ is referred to as the "size" of the RVE henceforth.

Furthermore, to better understand the role of the stiffness ratio, all numerical studies consider three stiffness ratios of $0.1,1$ and 10. The stiffness ratio incl./matr. $=0.1$ corresponds to the case where the inclusion is 10 times more compliant compared to the matrix. The significance of this case is that in the limit case of incl./matr. $\rightarrow 0$ the RVE recovers a porous medium rather than a composite. If both the inclusion and the matrix are identical, the stiffness ratio incl./matr. equals one. This specific case is important since it results in the most pronounced interface influence. Obviously, if the inclusion and the matrix are identical, the material response within the microstructure is uniform in the absence of interfaces. The stiffness 


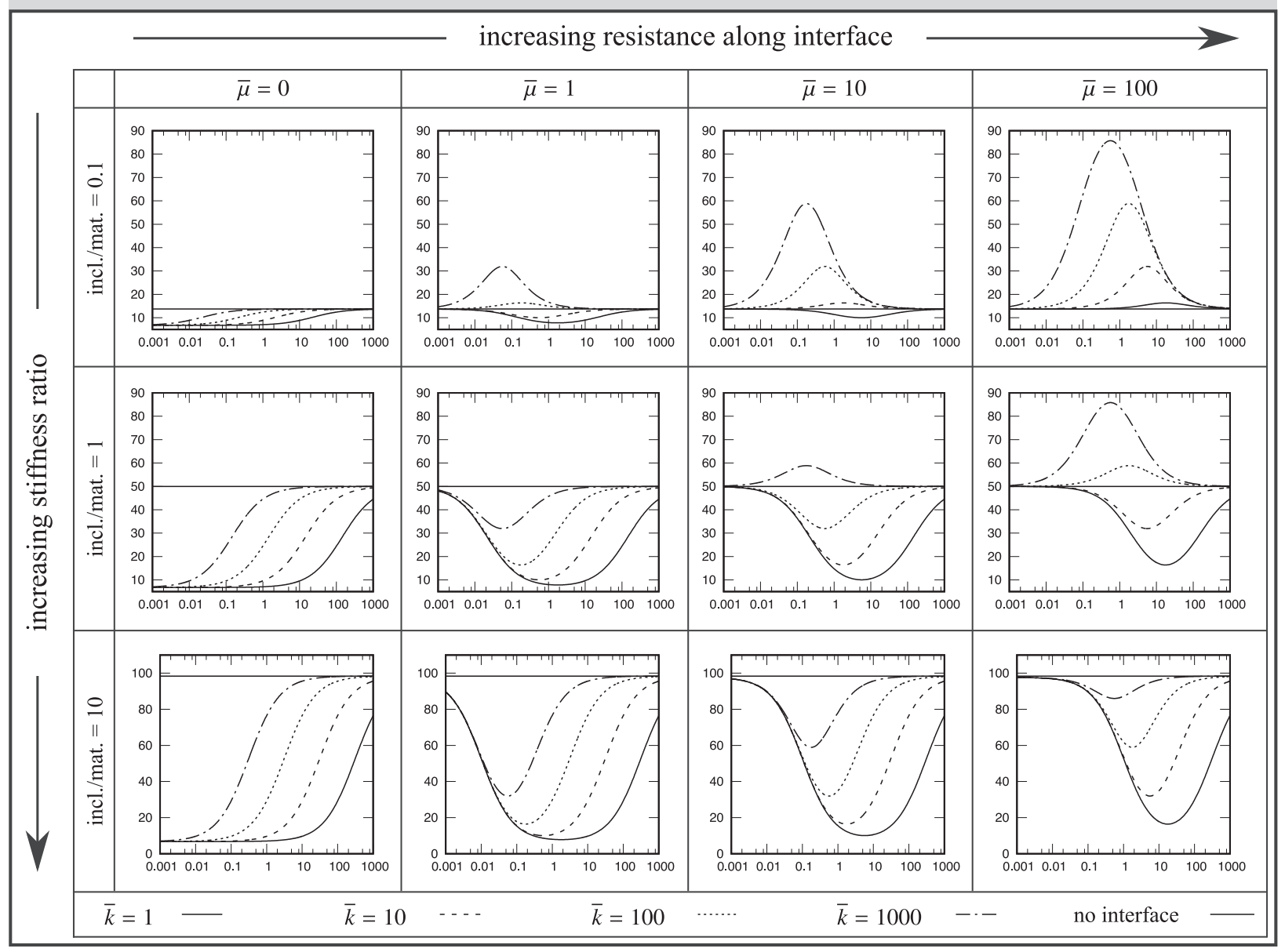

Fig. 10. Effective bulk modulus versus size of the RVE for the volume fraction of $50.26 \%$. Increasing the resistance against the change of length along the interface correlates to increasing the interface parameter $\bar{\mu}$. Different lines on each graph correspond to different orthogonal resistance of the interface against opening, see the legend at the bottom. In the absence of the interface, the size effect is completely ruled out resulting in a constant effective response.

ratio incl./matr. $=10$ corresponds to the case where the inclusion is 10 times stiffer than the matrix. The significance of this case is that in the limit case of incl./matr. $\rightarrow \infty$ the RVE recovers a matrix with rigid inclusions.

For all the examples, the material parameters of the matrix are constant and assumed to be $\mu=10$ and $\kappa=50$ with $\mu$ and $\kappa$ being the shear and bulk modulus, respectively. The stiffness ratio incl./matr. essentially identifies the material parameters of the fiber. Interface material parameters are varied to produce a wide spectrum of different cases. The inplane interface parameter varies from $\bar{\mu}=0$ indicating no in-plane resistance to $\bar{\mu}=100$ corresponding to a significant in-plane resistance of the interface. The orthogonal interface parameter varies from $\bar{k}=1$ to $\bar{k}=1000$. Note, the orthogonal interface parameter $\bar{k}$ indicates the resistance of the interface against opening. That is, in the limit of $\bar{k} \rightarrow \infty$ the interface remains coherent and cannot open due to the applied deformation. On the other hand, in the limit of $\bar{k} \rightarrow 0$ the interface shows almost no resistance against opening and hence, the fiber behaves completely detached from the matrix and does not influence the effective response, at all.

It shall be emphasized that here the numerical solution is understood as the "exact" effective response of the RVE and the analytical solution is an "approximate" one. The numerical solution is not altered by any assumption or approximation except those intrinsic to the finite element method itself. The finite element solution is accurate enough in the sense that it shows essentially no sensitivity with respect to further mesh refinement. The results are obtained for a relatively fine mesh with quadratic Lagrange finite elements. The details of the convergence tests are omitted for the sake of brevity.

The numerical examples are organized as follows. Figs. 8-13 provide a detailed numerical parametric study. The role of the interface on the overall behavior of the material is shown via several examples. Both the effective bulk and shear moduli are studied throughout the examples and for different volume fractions resulting in quantitatively as well as qualitatively different behavior. Figs. 14 and 15 provide a thorough comparison between the numerical and analytical solutions for both 


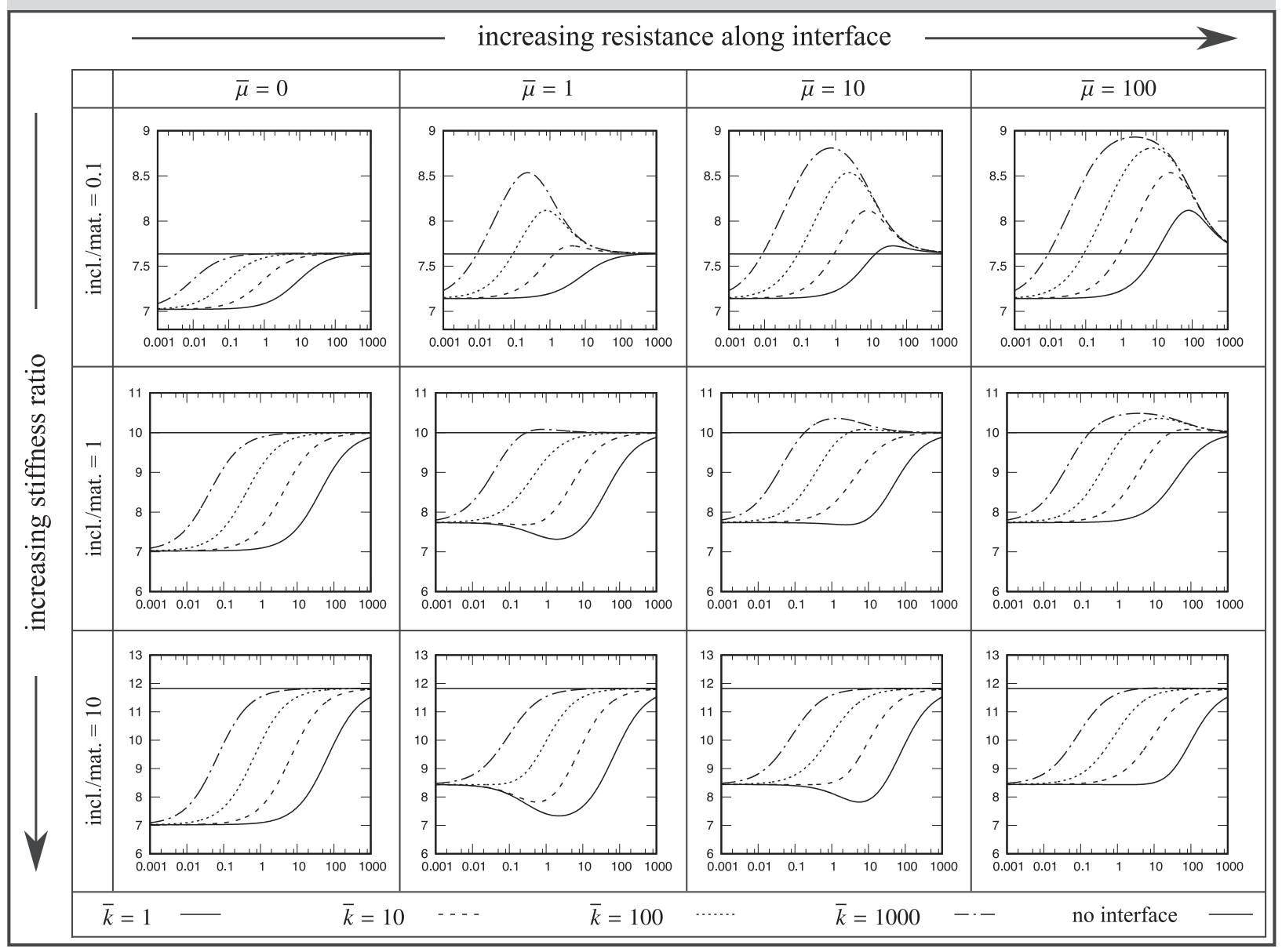

Fig. 11. Effective shear modulus versus size of the RVE for the volume fraction of $12.57 \%$. Increasing the resistance against the change of length along the interface correlates to increasing the interface parameter $\bar{\mu}$. Different lines on each graph correspond to different orthogonal resistance of the interface against opening, see the legend at the bottom. In the absence of the interface, the size effect is completely ruled out resulting in a constant effective response.

the effective bulk modulus and the effective shear modulus, respectively. It is concluded that the analytical solution is in good agreement with the numerical results.

Fig. 8 illustrates the effective bulk modulus versus size of the RVE for the volume fraction of $f=12.57 \%$. Each column corresponds to a different elastic resistance along the interface where the first column shows no resistance at all against the change of the length of the interface. In the presence of the interface, the size-dependent effective response is observed for all the cases. The flat line corresponds to the classical case with no interface at all and hence, no size effect. On the first column for a given stiffness ratio, reducing the size of the RVE results in a weaker response. This is expected since decreasing size results in more pronounced interface influence on the overall behavior. On the first column, the elastic resistance along the interface vanishes entirely and therefore, the interface behaves exactly according to the cohesive interface model resulting in the smaller-weaker behavior. On the second column, the overall behavior is rather complex and a combination of the elastic resistance along the interface as well as the cohesive one across the interface. For very large RVE, the influence of the interface almost vanishes and therefore, all the lines coincide asymptotically with the straight line of no interface effect. The effective bulk modulus shows a non-uniform depending on the stiffness ratio and the orthogonal resistance of the interface. More interestingly, in the presence of the elastic resistance along the interface, always a critical size exists at which the overall response reaches its extreme value. Furthermore, for a given set of interface parameters the critical size seems to be independent of the stiffness ratio. Figs. 9 and 10 overall result in the same conclusions and observations of Fig. 8 and only with minor differences. Finally, the difference between the minimum and maximum effective response for each case increases through increasing the volume fraction.

Figs. 11-13 illustrate the effective shear modulus versus size of the RVE for various volume fractions. Overall and qualitatively, all the three figures lead to identical conclusions with minor differences. Similar to the effective bulk modulus, in 


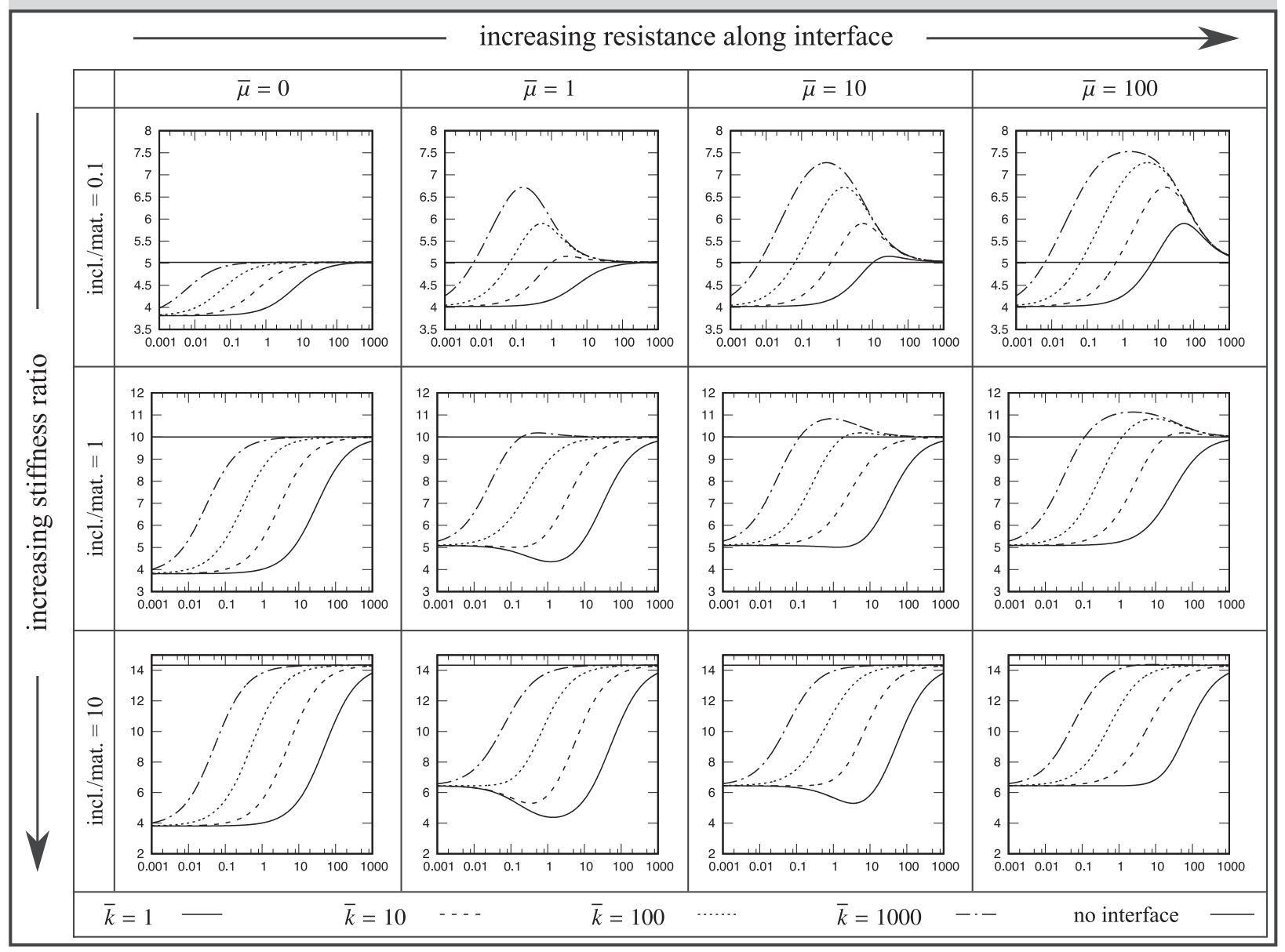

Fig. 12. Effective shear modulus versus size of the RVE for the volume fraction of $28.27 \%$. Increasing the resistance against the change of length along the interface correlates to increasing the interface parameter $\bar{\mu}$. Different lines on each graph correspond to different orthogonal resistance of the interface against opening, see the legend at the bottom. In the absence of the interface, the size effect is completely ruled out resulting in a constant effective response.

the presence of the interface a size effect is observed. Consequently, in the absence of the interface, the numerical studies furnish a flat line indicating no size effect, as expected. Analogous to the effective bulk modulus, on the first column for a given stiffness ratio, reducing the size of the RVE results in a weaker response. This can be justified by the fact that decreasing size results in more pronounced interface influence on the overall behavior. On the first column, the interface behaves merely according to the cohesive interface model resulting in the smaller-weaker trend. On all other columns, the overall behavior is rather complex as it combines both interface responses along and across the interface. Increasing the size of the RVE, diminishes the influence of the interface on the effective response and eventually vanishes altogether. That is, for very large RVE all the lines coincide asymptotically with the straight line of no interface effect. In contrast to the effective bulk modulus, a critical size for the RVE does not necessarily exist in all scenarios. As expected, the difference between the minimum and maximum effective response for each case increases through increasing the volume fraction.

Next, we compare the numerical results against the ones obtained via the analytical solution. The graphs in Fig. 14 and Fig. 15 correspond to the effective bulk modulus and shear modulus versus size, respectively. Overall, the analytical solutions are in very good agreement with the numerical ones. As expected, the analytical solution deviates more from the numerical one at higher volume fractions. Also, the analytical solution almost coincides with the numerical one for the effective bulk modulus. That is, the graphs of Fig. 14 show are nearly identical for both numerical and analytical approaches. Only at the highest volume fraction and for stiffness ratio of 0.1 , the error is noticeable. This is not the case for the effective shear modulus. In the case of shear, an error is observable for all volume fractions and material parameters. Nonetheless, the analytical solution captures the trend almost perfectly and only with a shift from the numerical solution. In other words, the analytical solution qualitatively resembles the numerical one but not quantitatively. This is particularly appealing since the graphs are neither uniform nor self-similar. Furthermore, in the presence of elastic resistance along the interface, the 


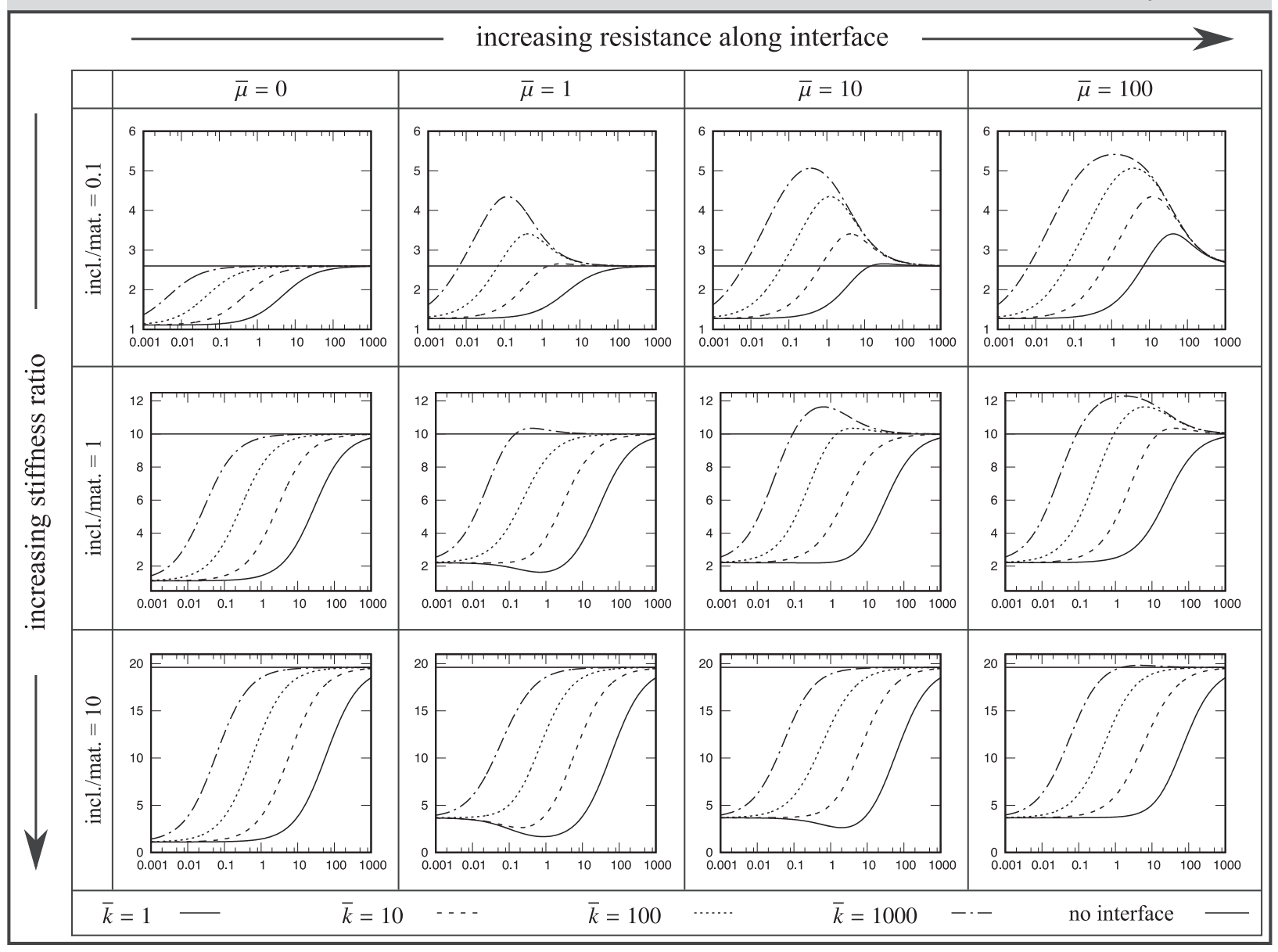

Fig. 13. Effective shear modulus versus size of the RVE for the volume fraction of $50.26 \%$. Increasing the resistance against the change of length along the interface correlates to increasing the interface parameter $\bar{\mu}$. Different lines on each graph correspond to different orthogonal resistance of the interface against opening, see the legend at the bottom. In the absence of the interface, the size effect is completely ruled out resulting in a constant effective response.

analytical solution is closer to the numerical one. This observation implies that including the elastic interface model results in a more accurate analytical estimate. This is a very interesting observation and can be further investigated in subsequent contributions.

\section{Conclusion and outlook}

Commonly accepted strategies to capture the interface behavior fall into two categories of the elastic and cohesive interfaces. Both the elastic and cohesive interface models can be unified as the limit cases of a broader general interface model. The governing equations for the general interface model at small-strain elasticity is given. Next, a first-order homogenization framework is established accounting for interfaces at the microscale whereby the interface represents the thin interphases between the fiber and the matrix. Obviously, unlike the classical first-order homogenization, the interface-enhanced homogenization here possesses a length-scale. Hence, the established methodology here captures the size effect in the overall behavior of continua. The length scale due to the general interface is more complex than the one due to either the elastic or cohesive interface models. The general interface model introduces two length scales into the problem. One is associated with the elastic resistance along the interface and roughly speaking results in the "smaller-stronger" effect. The other length scale is due to the cohesive nature of the interface that leads to the "smaller-weaker" effect. Obviously, the overall response of the general interface is a complex combination of both effects illustrated via the numerical examples. Using the composite cylinders approach, an analytical solution for the effective behavior of the composite is proposed. Through a series of numerical examples it is shown that the proposed analytical solution is in excellent agreement with the results obtained from the finite element method. Furthermore, it is observed that the size-dependent material response is bounded. That 


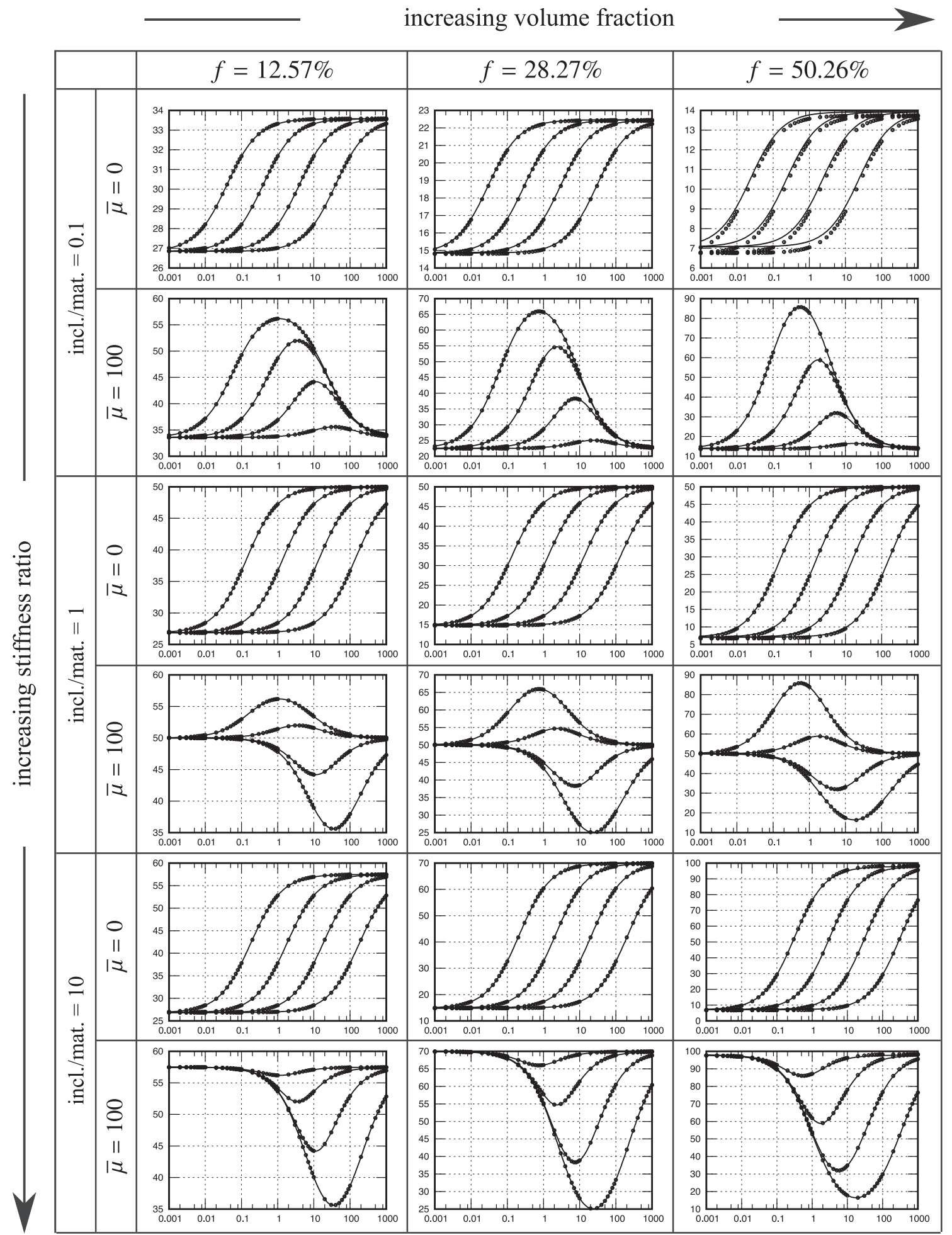

Fig. 14. Comparison between numerical and analytical solution of the effective bulk modulus versus size for various volume fractions and stiffness ratios. The lines and dots correspond to the analytical and numerical solution, respectively. 


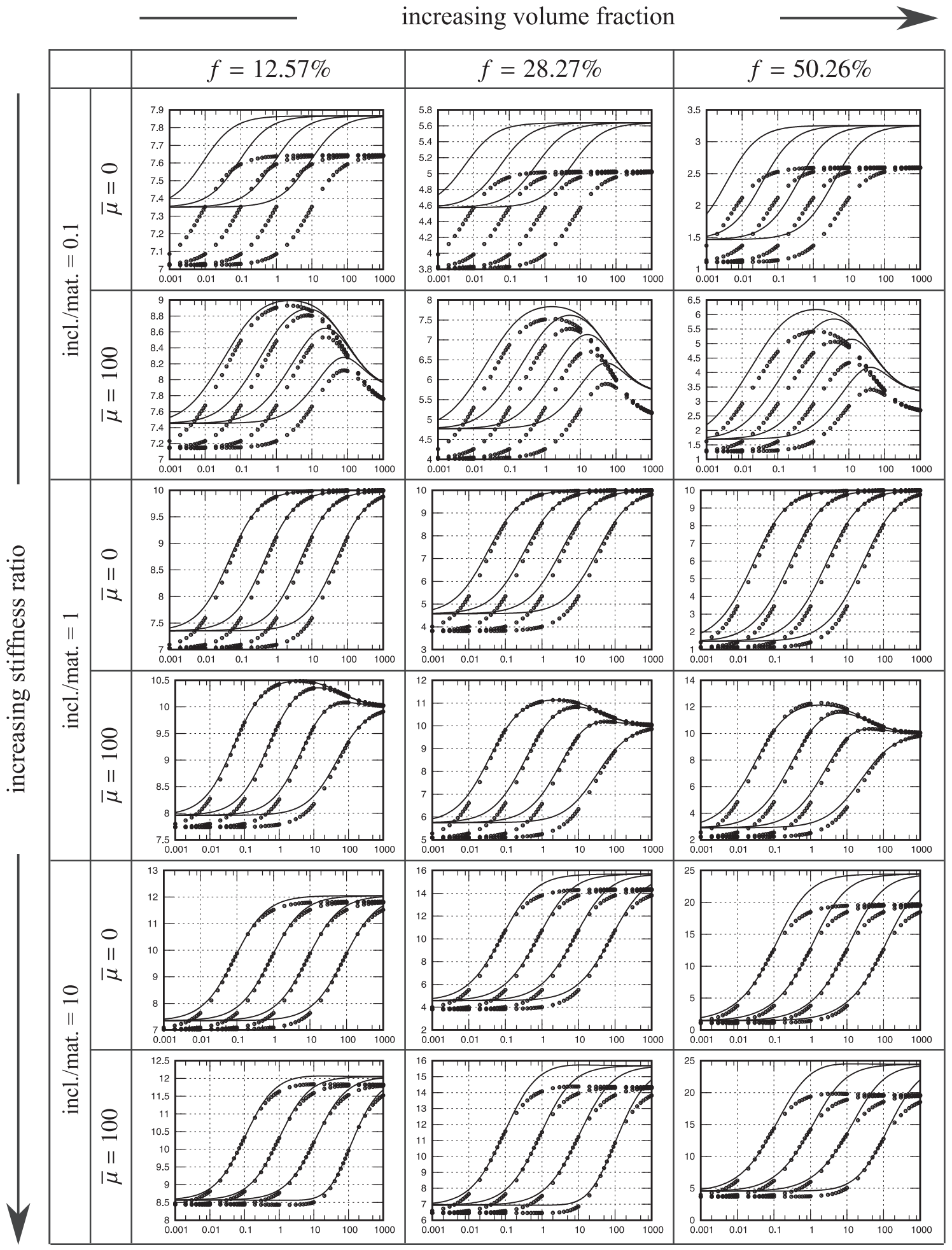

Fig. 15. Comparison between numerical and analytical solution of the effective shear modulus versus size for various volume fractions and stiffness ratios. The lines and dots correspond to the analytical and numerical solution, respectively. 
is, the size-dependent macro quantity of interest cannot exceed certain mathematical bounds. Obviously, the bounds are asymptotic in the sense that they indicate how much one can gain or lose at most for a given micro-structure. The next immediate extension of this work is to account for damage at the microscale and in particular, to take the cohesive damage of the interface into account. Another extension of the current contribution is to perform a parameter identification. More precisely, our ultimate goal is to estimate the interface constants from the size dependent overall response of the material. Such a parameter identification reveals whether or not the influence of the interface tension, curvature or eventually higher gradients on the overall material response is significant.

In summary, this manuscript presents our first attempt to shed light on comparing analytical and computational homogenization accounting for general interfaces. The proposed framework allows to predict a size effect in the material response missing in the classical first-order computational homogenization. This generic framework is broadly applicable to improve the understanding of the size-dependent behavior of continua with a large variety of applications in nano-materials.

\section{Appendix A. Generalized Young-Laplace equation}

The balance equation along the interface $(4)_{3}$ can be understood as a generalized Young-Laplace equation since it reduces to the classical Young-Laplace equation. In order to further clarify the balance Eq. $(4)_{3}$, the classical example of the YoungLaplace equation is investigated here. The Young-Laplace equation states that the balance equation on the interface between two static fluids reads

$$
\Delta p=\bar{\gamma}\left[\frac{1}{r_{1}}+\frac{1}{r_{2}}\right]
$$

where $\Delta p=p_{\text {in }}-p_{\text {out }}$ is the pressure difference across the fluid interface and $r_{1}$ and $r_{2}$ are the principal radii of curvature. The balance equation along the interface $(4)_{3}$ reads

$$
\overline{\operatorname{div}} \overline{\boldsymbol{\sigma}}+\llbracket \boldsymbol{\sigma} \rrbracket \cdot \overline{\boldsymbol{n}}=\mathbf{0}
$$

in which $\sigma$ and $\bar{\sigma}$ denote the bulk and interface stresses, respectively. Considering that $\llbracket \sigma \rrbracket=\sigma_{\text {out }}-\sigma_{\text {in }}$, and employing the relation between the hydrostatic pressure $p$ and stress $\boldsymbol{\sigma}$ for inviscid fluids $\boldsymbol{\sigma}=-p \boldsymbol{i}$, results in $\llbracket \boldsymbol{\sigma} \rrbracket=\Delta p \boldsymbol{i}$. Thus, the interface balance (A.2) reads

$$
\overline{\operatorname{div}} \overline{\boldsymbol{\sigma}}+\Delta p \boldsymbol{i} \cdot \overline{\boldsymbol{n}}=\mathbf{0} \quad \Rightarrow \quad \Delta p \overline{\boldsymbol{n}}=-\overline{\operatorname{div}} \overline{\boldsymbol{\sigma}} .
$$

Inserting the interface stress $\overline{\boldsymbol{\sigma}}=\bar{\gamma} \overline{\boldsymbol{i}}$ due to surface-tension $\bar{\gamma}$ into the interface balance (A.3) yields

$$
\Delta p \overline{\boldsymbol{n}}=-\overline{\operatorname{div}}(\bar{\gamma} \overline{\boldsymbol{i}})=-\bar{\gamma} \overline{\operatorname{div}} \overline{\boldsymbol{i}}=-\bar{\gamma} \bar{\kappa} \overline{\boldsymbol{n}} \quad \Rightarrow \quad \Delta p=-\bar{\gamma} \bar{\kappa},
$$

in which the identity $\overline{\operatorname{div}} \overline{\boldsymbol{i}}=-[\overline{\operatorname{div}} \overline{\boldsymbol{n}}] \overline{\boldsymbol{n}}=\bar{\kappa} \overline{\boldsymbol{n}}$ is used. To obtain the Young-Laplace equation in its classical format, the geometrical definition of the interface curvature

$$
\bar{\kappa}=-\left[\frac{1}{r_{1}}+\frac{1}{r_{2}}\right]
$$

is required in which the negative sign arises from the convention that the curvature is negative if the interface curves away from its normal, and that the radii of curvatures are always positive. This examples explains how the curvature is embedded within the interface divergence operator.

\section{Appendix B. In-plane shear modulus}

Similar to the effective bulk modulus, it is possible to compute the effective shear modulus for available interface types via simplification of the current (general) interface model as

- Perfect interface model can be recovered by setting $\bar{\mu}=0$ and $\bar{k} \rightarrow \infty$,

- Cohesive interface model can be recovered by setting $\bar{\mu}=0$ and $\bar{k} \nrightarrow \infty$,

- Elastic interface model can be recovered by setting $\bar{\mu} \neq 0$ and $\bar{k} \rightarrow \infty$,

- General interface model can be recovered by setting $\bar{\mu} \neq 0$ and $\bar{k} \nrightarrow \infty$.

For a unidirectional fiber composite presenting generalized interfaces between the matrix and the fibers, the macroscopic in-plane shear modulus is the positive root of the polynomial

$$
A^{\mathrm{M}} \mu^{2}+B{ }^{\mathrm{M}} \mu+C=0 \text { with }\left\{\begin{array}{l}
A=4 e_{a}+4 e_{b} g_{b}+4 f_{a} r_{2}^{2}+4 f_{b} g_{b} r_{2}^{2}+4 d_{a} r_{2}^{4}+4 d_{b} g_{b} r_{2}^{4}+4 c_{a} r_{2}^{6}+4 c_{b} g_{b} r_{2}^{6}, \\
B=4 e_{b} g_{a}+4 f_{b} g_{a} r_{2}^{2}-2 r_{2}^{4}+4 d_{b} g_{a} r_{2}^{4}+g_{b} r_{2}^{4}+4 c_{b} g_{a} r_{2}^{6}, \\
C=g_{a} r_{2}^{4},
\end{array}\right.
$$

in which

$$
g_{a}=\frac{r_{2}}{\frac{f_{b}}{r_{2}}+\frac{f_{b}\left[\lambda^{(2)}+2 \mu^{(2)}\right]}{\mu^{(2)} r_{2}}+2 d_{b} r_{2}+c_{b} r_{2}^{3}+\frac{c_{b} \lambda^{(2)} r_{2}^{3}}{2 \lambda^{(2)}+3 \mu^{(2)}}},
$$


and

$$
g_{b}=-\frac{\frac{f_{a}}{r_{2}}+\frac{f_{a}\left[\lambda^{(2)}+2 \mu^{(2)}\right]}{\mu^{(2)} r_{2}}+2 d_{a} r_{2}+c_{a} r_{2}^{3}+\frac{c_{a} \lambda^{(2)} r_{2}^{3}}{2 \lambda^{(2)}+3 \mu^{(2)}}}{\frac{f_{b}}{r_{2}}+\frac{f_{b}\left[\lambda^{(2)}+2 \mu^{(2)}\right]}{\mu^{(2)} r_{2}}+2 d_{b} r_{2}+c_{b} r_{2}^{3}+\frac{c_{b} \lambda^{(2)} r_{2}^{3}}{2 \lambda^{(2)}+3 \mu^{(2)}}} .
$$

The parameters $c_{a}, c_{b}, d_{a}, d_{b}, e_{a}, e_{b}, f_{a}, f_{b}$, are provided from the solution of the linear system

$$
\boldsymbol{A U}=\boldsymbol{F} \text { with } \boldsymbol{A}=\left[\begin{array}{cccccc}
A_{11} & A_{12} & A_{13} & A_{14} & A_{15} & A_{16} \\
A_{21} & A_{22} & A_{23} & A_{24} & A_{25} & A_{26} \\
A_{31} & A_{32} & A_{33} & A_{34} & A_{35} & A_{36} \\
A_{41} & A_{42} & A_{43} & A_{44} & A_{45} & A_{46} \\
0 & 0 & 0 & A_{54} & A_{55} & A_{56} \\
0 & 0 & A_{63} & A_{64} & A_{65} & A_{66}
\end{array}\right], \quad \boldsymbol{U}=\left[\begin{array}{cc}
a_{a} & a_{b} \\
b_{a} & b_{b} \\
c_{a} & c_{b} \\
d_{a} & d_{b} \\
e_{a} & e_{b} \\
f_{a} & f_{b}
\end{array}\right], \quad \boldsymbol{F}=\left[\begin{array}{cc}
0 & 0 \\
0 & 0 \\
0 & 0 \\
0 & 0 \\
1 & -1.5 \\
1 & 1.5
\end{array}\right] .
$$

The components of $\boldsymbol{A}$ are obtained through the relations

$$
\begin{aligned}
& A_{11}=\frac{\lambda^{(1)} r_{1}^{3}}{2 \lambda^{(1)}+3 \mu^{(1)}}, \\
& A_{13}=-\frac{\lambda^{(2)} r_{1}^{3}}{2 \lambda^{(2)}+3 \mu^{(2)}} \text {, } \\
& A_{15}=-A_{25}=\frac{3 \mu^{(2)}}{\bar{k} r_{1}^{4}}+\frac{1}{r_{1}^{3}} \text {, } \\
& A_{21}=\frac{3\left[\lambda^{(1)}+\mu^{(1)}\right] \mu^{(1)} r_{1}^{2}}{\bar{k}\left[2 \lambda^{(1)}+3 \mu^{(1)}\right]}+r_{1}^{3} \text {, } \\
& A_{26}=\frac{\lambda^{(2)}+\mu^{(2)}}{\bar{k} r_{1}^{2}}-\frac{1}{r_{1}} \\
& A_{32}=-2 \mu^{(1)}+\frac{\bar{\mu}}{r_{1}}, \\
& A_{34}=2 \mu^{(2)}+\frac{\bar{\mu}}{r_{1}}, \\
& A_{36}=\frac{\mu^{(2)} \bar{\mu}-\left[\lambda^{(2)}+\mu^{(2)}\right]\left[\bar{\mu}+4 \mu^{(2)} r_{1}\right]}{\mu^{(2)} r_{1}^{3}} \text {, } \\
& A_{42}=-\frac{2\left[\bar{\mu}+\mu^{(1)} r_{1}\right]}{r_{1}} \text {, } \\
& A_{44}=-\frac{2\left[\bar{\mu}-\mu^{(2)} r_{1}\right]}{r_{1}}, \\
& A_{46}=\frac{2\left[2 \lambda^{(1)}+3 \mu^{(1)}\right]\left[-\mu^{(2)} \bar{\mu}+\left[\lambda^{(2)}+\mu^{(2)}\right]\left[\bar{\mu}+\mu^{(2)} r_{1}\right]\right]}{\left[2 \lambda^{(1)}+3 \mu^{(1)}\right] \mu^{(2)} r_{1}^{3}} \\
& A_{55}=-A_{65}=\frac{6 \mu^{(2)}}{r_{2}^{4}}, \quad A_{56}=-2 A_{66}=-\frac{4\left[\lambda^{(2)}+\mu^{(2)}\right]}{r_{2}^{2}}, \quad A_{63}=\frac{6\left[\lambda^{(2)}+\mu^{(2)}\right] \mu^{(2)} r_{2}^{2}}{2 \lambda^{(2)}+3 \mu^{(2)}} \\
& A_{12}=A_{22}=\frac{\mu^{(1)}}{\bar{k}}+r_{1}, \\
& A_{14}=A_{24}=\frac{\mu^{(2)}}{\bar{k}}-r_{1}, \\
& A_{16}=-\frac{2 \lambda^{(2)}+2 \mu^{(2)}}{\bar{k} r_{1}^{2}}-\frac{\lambda^{(2)}+2 \mu^{(2)}}{\mu^{(2)} r_{1}}, \\
& A_{23}=\frac{3\left[\lambda^{(2)}+\mu^{(2)}\right] \mu^{(2)} r_{1}^{2}}{\bar{k}\left[2 \lambda(2)+3 \mu^{(2)}\right]}-r_{1}^{3} \\
& A_{31}=\frac{3\left[\lambda^{(1)}+2 \mu^{(1)}\right] \bar{\mu} r_{1}}{2 \lambda(1)+3 \mu^{(1)}} \text {, } \\
& A_{33}=\frac{3\left[\lambda^{(2)}+2 \mu^{(2)}\right] \bar{\mu} r_{1}}{2 \lambda^{(2)}+3 \mu^{(2)}} \text {, } \\
& A_{35}=\frac{3\left[\bar{\mu}+2 \mu^{(2)} r_{1}\right]}{r_{1}^{5}} \text {, } \\
& A_{41}=-\frac{6 r_{1}\left[\left[\lambda^{(1)}+2 \mu^{(1)}\right] \bar{\mu}+\left[\lambda^{(1)}+\mu^{(1)}\right] \mu^{(1)} r_{1}\right]}{2 \lambda^{(1)}+3 \mu^{(1)}}, \\
& A_{43}=-\frac{6 r_{1}\left[\left[\lambda^{(2)}+2 \mu^{(2)}\right] \bar{\mu}-\left[\lambda^{(2)}+\mu^{(2)}\right] \mu^{(2)} r_{1}\right]}{2 \lambda^{(2)}+3 \mu^{(2)}}, \\
& A_{45}=-\frac{6\left[\bar{\mu}-\mu^{(2)} r_{1}\right]}{r_{1}^{5}}, \\
& A_{54}=A_{64}=2 \mu^{(2)} \text {, }
\end{aligned}
$$

\section{Appendix C. Further elastic constants}

\section{C1. Axial shearing}

To identify the axial shear modulus, the RVE of the composite is subjected to shear displacement in the direction parallel to the fiber, while the top and bottom edges have zero normal stress, as shown in Fig. C.16. Under these conditions, the displacement is zero at the $r$ and $\theta$ directions, while the displacement field in the $z$ direction is expressed as

$$
u_{z}^{(i)}=\left[D_{1}^{(i)} r+D_{2}^{(i)} \frac{1}{r}\right] \cos \theta, \quad i=1,2 .
$$

The strains and stresses for the fiber, the matrix and the general interface are expressed with the help of the relations (18)-(21). Analogous to the other elastic constants, the problem is reduced on calculating $D_{1}^{(1)}, D_{2}^{(1)}, D_{1}^{(2)}$ and $D_{2}^{(2)}$. These constants are obtained with the help of the overall boundary and compatibility conditions, as well as the jump conditions at the interface: 


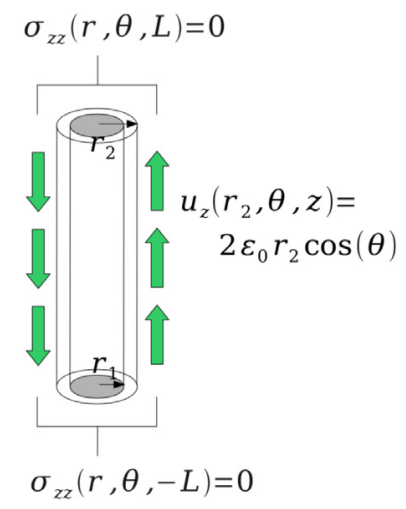

Fig. C.16. Fiber composite RVE under axial shear strain conditions.

- Finite solution at the center of the fiber

$$
u_{z} \text { finite at } r=0 \text { or } D_{2}^{(1)}=0 .
$$

- Boundary condition at the outer cylinder

$$
u_{z}^{(2)}\left(r_{2}\right)=2 \varepsilon_{0} r_{2} \cos \theta \text {. }
$$

- Traction equilibrium at the interface

$$
\frac{1}{r_{1}} \frac{\partial \bar{\sigma}_{\theta z}}{\partial \theta}+\sigma_{r z}^{(2)}\left(r_{1}\right)-\sigma_{r z}^{(1)}\left(r_{1}\right)=0
$$

- The average traction at the interface

$$
\frac{\sigma_{r z}^{(2)}\left(r_{1}\right)+\sigma_{r z}^{(1)}\left(r_{1}\right)}{2}=\bar{k}\left[u_{z}^{(2)}\left(r_{1}\right)-u_{z}^{(1)}\left(r_{1}\right)\right] .
$$

The system of Eqs. (C.1)-(C.4) is linear and can be solved explicitly, permitting to obtain the stresses, strains and displacements at all phases (matrix, fiber, interface). The macroscopic axial shear modulus is then computed according to the formula

$$
{ }^{\mathrm{M}} \mu_{\text {axial }}=\frac{\sigma_{r z}^{(2)}\left(r_{2}\right)}{2 \varepsilon_{0} \cos \theta},
$$

resulting in the analytical expression

$$
{ }^{\mathrm{M}} \mu_{\text {axial }}=\mu^{(2)} \frac{\left[\mu^{(1)}[1+c]+\mu^{(2)}[1-c]\right][1+\omega / \phi]+2 \mu^{(1)} \mu^{(2)}[1-c] / \phi+2[1+c] \omega}{\left[\mu^{(1)}[1-c]+\mu^{(2)}[1+c]\right][1+\omega / \phi]+2 \mu^{(1)} \mu^{(2)}[1+c] / \phi+2[1-c] \omega},
$$

with $\omega=\bar{\mu} / 2 r_{1}$ and $\phi=2 \bar{k} r_{1}$. For perfect interface the above expression is reduced to the one provided by Christensen and Lo (1979).

\section{C2. Axial Young's modulus and stiffness coefficient}

The axial Young's modulus and the axial stiffness coefficient are computed with the help of similar boundary value problems illustrated in Fig. C.17. The RVE of the composite is subjected to uniaxial stretching along the fiber direction, while circumferentially it is considered either traction free (for the Young's modulus) or displacement fixed (for the axial stiffness component). Under such configuration the displacement fields are given by the expressions

$$
u_{r}^{(i)}=D_{1}^{(i)} r+D_{2}^{(i)} \frac{1}{r}, \quad u_{z}^{(i)}=\varepsilon_{0} z, \quad i=1,2,
$$

where the coefficients $D$ are constants to be evaluated.

The strains and stresses for the fiber, the matrix and the general interface are expressed with the help of the relations (18)-(21). The equations of these two problems are the following:

- Finite solution at the center of the fiber

$$
u_{z} \text { finite at } r=0 \text { or } \quad D_{2}^{(1)}=0 .
$$

- Boundary condition at the outer cylinder

$$
\sigma_{r r}^{(2)}\left(r_{2}\right)=0 \text { (axial Young's modulus), } \quad u_{r}^{(2)}\left(r_{2}\right)=0 \text { (axial stiffness coefficient) . }
$$




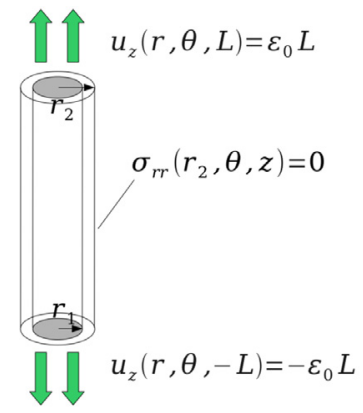

(a)

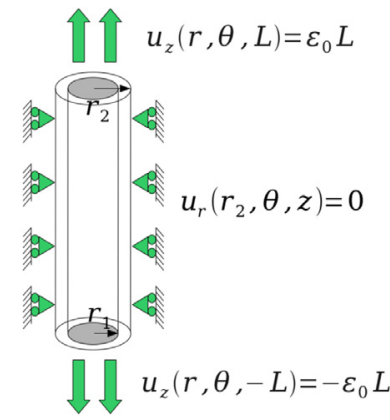

(b)

Fig. C.17. Boundary value problems for a) axial Young's modulus and b) axial stiffness coefficient.

- Traction equilibrium at the interface

$$
-\frac{\bar{\sigma}_{\theta \theta}}{r_{1}}+\sigma_{r r}^{(2)}\left(r_{1}\right)-\sigma_{r r}^{(1)}\left(r_{1}\right)=0 .
$$

- The average traction at the interface

$$
\frac{\sigma_{r r}^{(2)}\left(r_{1}\right)+\sigma_{r r}^{(1)}\left(r_{1}\right)}{2}=\bar{k}\left[u_{r}^{(2)}\left(r_{1}\right)-u_{r}^{(1)}\left(r_{1}\right)\right] .
$$

From this system of equations the stresses, strains and displacements at all phases (matrix, fiber, interface) are computed, allowing to evaluate the average mechanical work in the RVE. Then both elastic constants are given by the formula Chatzigeorgiou et al. (2012)

$$
{ }^{\mathrm{M}} E_{z}=\frac{2}{\mathscr{V} \varepsilon_{0}^{2}} \int_{\mathcal{B}} W \mathrm{~d} V \text { for } \mathrm{BC}(C .8)_{1}, \quad{ }^{\mathrm{M}} C_{z z z z}=\frac{2}{\mathscr{V} \varepsilon_{0}^{2}} \int_{\mathcal{B}} W \mathrm{~d} V \quad \text { for } \mathrm{BC}(C .8)_{2},
$$

resulting in the analytical expressions

$$
\begin{aligned}
& { }^{\mathrm{M}} E_{z}=\left[\left[\lambda^{(1)}\left[1+A_{1}\right]+2 \mu^{(1)}\right] c+\left[\lambda^{(2)}\left[1+B_{1}\right]+2 \mu^{(2)}\right][1-c]\right]+8 c \omega, \\
& { }^{\mathrm{M}} C_{z z z z}=\left[\left[\lambda^{(1)}\left[1+A_{2}\right]+2 \mu^{(1)}\right] c+\left[\lambda^{(2)}\left[1+B_{2}\right]+2 \mu^{(2)}\right][1-c]\right]+8 c \omega,
\end{aligned}
$$

in which

$$
\begin{aligned}
A_{1}= & \frac{a_{11}+a_{12} \omega / \phi}{a_{13}+a_{14} \omega / \phi}, \quad B_{1}=\frac{c \mu^{(2)} b_{11}-\lambda^{(2)} b_{12}}{c \mu^{(2)} b_{13}+\left[\lambda^{(2)}+\mu^{(2)}\right] b_{12}}, \\
A_{2}= & \frac{a_{21}}{a_{22}+a_{23} / \phi}, \quad B_{2}=\frac{c b_{11}}{c b_{13}-b_{12}}, \\
a_{11}= & c \mu^{(2)}\left[\lambda^{(1)}-\lambda^{(2)}\right]+\lambda^{(2)} \mu^{(2)}+\lambda^{(1)}\left[\lambda^{(2)}+\mu^{(2)}\right]+2 \lambda^{(1)} \mu^{(2)}\left[\lambda^{(2)}+\mu^{(2)}\right][1-c] / \phi, \\
a_{12}= & c \mu^{(2)}\left[\lambda^{(2)}+\lambda^{(1)}\right]+\lambda^{(1)}\left[\lambda^{(2)}+\mu^{(2)}\right]-\lambda^{(2)} \mu^{(2)}, \\
a_{13}= & -2\left[\lambda^{(1)}+\mu^{(1)}\right] \mu^{(2)}\left[\lambda^{(2)}+\mu^{(2)}\right][1-c] / \phi-\mu^{(2)}\left[\lambda^{(2)}+\mu^{(2)}\right][1-c] \\
& -\left[\lambda^{(2)}+\mu^{(2)}[1+c]\right]\left[\lambda^{(1)}+\mu^{(1)}+2 \omega\right], \\
a_{14}= & c \mu^{(2)}\left[\lambda^{(1)}+\mu^{(1)}-\lambda^{(2)}-\mu^{(2)}\right]+\left[\lambda^{(2)}+\mu^{(2)}\right]\left[\lambda^{(1)}+\mu^{(1)}+\mu^{(2)}\right], \\
b_{11}= & \lambda^{(2)}-\lambda^{(1)}+2 \lambda^{(2)}\left[\lambda^{(1)}+\mu^{(1)}\right] / \phi+\left[\lambda^{(1)}+\lambda^{(2)}\right] \omega / \phi, \\
b_{12}= & 2\left[\lambda^{(1)}+\mu^{(1)}\right] \mu^{(2)} / \phi+\left[\lambda^{(1)}+\mu^{(1)}+\mu^{(2)}\right][1+\omega / \phi]+2 \omega, \\
b_{13}= & -\left[1+\left[\lambda^{(1)}+\mu^{(1)}\right] / \phi\right]\left[\lambda^{(2)}+\mu^{(2)}-\omega\right]+\left[1-\left[\lambda^{(2)}+\mu^{(2)}\right] / \phi\right]\left[\lambda^{(1)}+\mu^{(1)}+\omega\right], \\
a_{21}= & -2 \lambda^{(1)}\left[c \lambda^{(2)}+[1+c] \mu^{(2)}\right] / \phi+\left[\lambda^{(2)}-\lambda^{(1)}\right][1-c]-\left[\lambda^{(1)}+\lambda^{(2)}\right][1-c] \omega / \phi, \\
a_{22}= & c\left[\lambda^{(2)}+\mu^{(2)}-\mu^{(1)}\right]+\mu^{(1)}+\mu^{(2)}+\left[\lambda^{(1)}+2 \omega\right][1-c], \\
a_{23}= & 2\left[\lambda^{(1)}+\mu^{(1)}\right]\left[\left[\lambda^{(2)}+\mu^{(2)}\right] c+\mu^{(2)}\right]+\left[\lambda^{(1)}+\mu^{(1)}+\mu^{(2)}-c\left[\lambda^{(1)}+\mu^{(1)}-\lambda^{(2)}-\mu^{(2)}\right]\right] \omega,
\end{aligned}
$$

with $\omega=\bar{\mu} / 2 r_{1}$ and $\phi=\bar{k} r_{1}$. 


\section{References}

Alfano, G., Crisfield, M.A., 2001. Finite element interface models for the delamination analysis of laminated composites: mechanical and computational issues. Int.J. Numer. Meth. Eng. 50, 1701-1736.

Altenbach, H., Eremeyev, V.A., 2011. On the shell theory on the nanoscale with surface stresses. Int. J. Eng. Sci. 49, $1294-1301$.

Barenblatt, G.I., 1959. The formation of equilibrium cracks during brittle fracture. general ideas and hypotheses. axially-symmetric cracks. J. Appl. Math. Mech. 23, 622-636.

Barenblatt, G.I., 1962. The mathematical theory of equilibrium cracks in brittle fracture. Adv. Appl. Mech. 7, 55-129.

Benveniste, Y., 2006. A general interface model for a three-dimensional curved thin anisotropic interphase between two anisotropic media. J. Mech. Phys. Solids $54,708-734$

Benveniste, Y., 2013. Models of thin interphases and the effective medium approximation in composite media with curvilinearly anisotropic coated inclusions. Int. J. Eng. Sci. 72, 140-154.

Benveniste, Y., 2013. Models of thin interphases with variable moduli in plane-strain elasticity. Math. Mech. Solids 18, 119-134

Benveniste, Y., Miloh, T., 2001. Imperfect soft and stiff interfaces in two-dimensional elasticity. Mech. Mater. 33, 309-323.

Benveniste, Y., Milton, G.W., 2010. The effective medium and the average field approximations vis-a-vis the Hashin-Shtrikman bounds. I. the self-consistent scheme in matrix-based composites. J. Mech. Phys. Solids 58, 1026-1038.

Benveniste, Y., Milton, G.W., 2010. The effective medium and the average field approximations vis-a-vis the Hashin-Shtrikman bounds. II. the generalized self-consistent scheme in matrix-based composites. J. Mech. Phys. Solids 58, 1039-1056.

Bövik, P., 1994. On the modelling of thin interface layers in elastic and acoustic scattering problems. Q. J. Mech. Appl. Math. 47, 17-42.

van den Bosch, M.J., Schreurs, P.J.G., Geers, M.G.D., 2006. An improved description of the exponential xu and needleman cohesive zone law for mixed-mode decohesion. Eng. Fract. Mech. 73, 1220-1234.

Brisard, S., Dormieux, L., Kondo, D., 2010. Hashin-shtrikman bounds on the shear modulus of a nanocomposite with spherical inclusions and interface effects. Comput. Mater. Sci. 50, 403-410.

Charlotte, M., Laverne, J., Marigo, J.J., 2006. Initiation of cracks with cohesive force models: a variational approach. Eur. J. Mech. Solids 25, 649-669.

Chatzigeorgiou, G., Javili, A., Steinmann, P., 2015. Multiscale modelling for composites with energetic interfaces at the micro- or nanoscale. Math. Mech. Solids 20, 1130-1145.

Chatzigeorgiou, G., Seidel, G.D., Lagoudas, D.C., 2012. Effective mechanical properties of aligned "fuzzy fiber" composites. Composites Part B 43, $2577-2593$.

Chen, T., Chiu, M.S., Weng, C.N., 2006. Derivation of the generalized young-laplace equation of curved interfaces in nanoscaled solids. J. Appl. Phys. 100, 074308.

Chen, Y., Zhang, Z., Huang, R., Huang, Z.P., 2016. Effect of residual interface stress on thermo-elastic properties of unidirectional fiber-reinforced nanocomposites. Int. J. Mech. Sci. 113, 133-147.

Chhapadia, P., Mohammadi, P., Sharma, P., 2011. Curvature-dependent surface energy and implications for nanostructures. J. Mech. Phys. Solids 59, 2103-2115.

Christensen, R., Lo, K., 1979. Solutions for effective shear properties in three phase sphere and cylinder models. J. Mech. Phys. Solids $27,315-330$.

Christensen, R.M., 1979. Mechanics of composite materials. Dover, New York, USA.

Cordero, N.M., Forest, S., Busso, E.P., 2016. Second strain gradient elasticity of nano-objects. J. Mech. Phys. Solids 97, 92-124.

Daher, N., Maugin, G.A., 1986. The method of virtual power in continuum mechanics application to media presenting singular surfaces and interfaces. Acta Mech. 60, 217-240.

Davydov, D., Javili, A., Steinmann, P., 2013. On molecular statics and surface-enhanced continuum modeling of nano-structures. Comput. Mater. Sci. 69, $510-519$.

Dell'Isola, F., Romano, A., 1987. On the derivation of thermomechanical balance equations for continuous systems with a nonmaterial interface. Int. J. Eng. Sci. 25, 1459-1468.

Despringre, N., Chemisky, Y., Bonnay, K., Meraghni, F., 2016. Micromechanical modeling of damage and load transfer in particulate composites with partially debonded interface. Compos. Struct. 155, 77-88.

Dimitri, R., Trullo, M., De Lorenzis, L., Zavarise, G., 2015. Coupled cohesive zone models for mixed-mode fracture: a comparative study. Eng. Fract. Mech. $148,145-179$.

Dingreville, R., Hallil, A., Berbenni, S., 2014. From coherent to incoherent mismatched interfaces: a generalized continuum formulation of surface stresses. J. Mech. Phys. Solids 72, 40-60.

Dingreville, R., Qu, J., 2008. Interfacial excess energy, excess stress and excess strain in elastic solids: planar interfaces. J. Mech. Phys. Solids 56, $1944-1954$.

Duan, H.L., Karihaloo, B., 2007. Effective thermal conductivities of heterogeneous media containing multiple imperfectly bonded inclusions. Phys. Rev. B 75, $1-9$.

Duan, H.L., Wang, J., Huang, Z.P., Karihaloo, B.L., 2005. Eshelby formalism for nano-inhomogeneities. Proc. R. Soc. A 461, $3335-3353$.

Duan, H.L., Wang, J., Huang, Z.P., Karihaloo, B.L., 2005. Size-dependent effective elastic constants of solids containing nano-inhomogeneities with interface stress. J. Mech. Phys. Solids 53, 1574-1596.

Duan, H.L., Wang, J., Karihaloo, B.L., 2009. Theory of elasticity at the nanoscale. Adv. Appl. Mech. 42, 1-68.

Duan, H.L., Wang, J., Karihaloo, B.L., Huang, Z.P., 2006. Nanoporous materials can be made stiffer than non-porous counterparts by surface modification. Acta Materialia 54, 2983-2990.

Duan, H.L., Yi, X., Huang, Z.P., Wang, J., 2007. A unified scheme for prediction of effective moduli of multiphase composites with interface effects. part I: theoretical framework. Mech. Mater. 39, 81-93.

Dugdale, D., 1960. Yielding of steel sheets containing slits. J. Mech. Phys. Solids 8, 100-104.

Elsner, B., Müller, S., Bargmann, S., Weissmüller, J., 2017. Surface excess elasticity of gold: ab initio coefficients and impact on the effective elastic response of nanowires. Acta Materialia 124, 468-477.

Fagerström, M., Larsson, R., 2006. Theory and numerics for finite deformation fracture modelling using strong discontinuities. Int. J. Numer. Methods Eng. 66, 911-948.

Fried, E., Gurtin, M.E., 2007. Thermomechanics of the interface between a body and its environment. Continuum Mech. Thermodyn. 19, 253-271.

Fried, E., Todres, R.E., 2005. Mind the gap: the shape of the free surface of a rubber-like material in proximity to a rigid contactor. J. Elast. 80, $97-151$.

Fritzen, F., Leuschner, M., 2015. Nonlinear reduced order homogenization of materials including cohesive interfaces. Comput. Mech. 56, 131-151.

Gao, X., Huang, Z.P., Fang, D., 2017. Curvature-dependent interfacial energy and its effects on the elastic properties of nanomaterials. Int. J. Solids Struct 100-107. 113114

Gao, X., Huang, Z.P., Qu, J., Fang, D., 2014. A curvature-dependent interfacial energy-based interface stress theory and its applications to nano-structured materials: (i) general theory. J. Mech. Phys. Solids 66, 59-77.

Gasser, T.C., Holzapfel, G.A., 2003. Geometrically non-linear and consistently linearized embedded strong discontinuity models for 3d problems with an application to the dissection analysis of soft biological tissues. Comput. Methods Appl. Mech. Eng. 192, 5059-5098.

Geers, M.G.D., Kouznetsova, V.G., Brekelmans, W.A.M., 2010. Multi-scale computational homogenization: trends and challenges. J. Comput. Appl. Math. 234, 2175-2182.

Gu, S.T., He, Q.C., 2011. Interfacial discontinuity relations for coupled multifield phenomena and their application to the modeling of thin interphases as imperfect interfaces. J. Mech. Phys. Solids 59, 1413-1426.

Gu, S.T., Monteiro, E., He, Q.C., 2011. Coordinate-free derivation and weak formulation of a general imperfect interface model for thermal conduction in composites. Compos. Sci. Technol. 71, 1209-1216. 
Gu, S.-T., Liu, J.-T., He, Q.-C., 2014. Size-dependent effective elastic moduli of particulate composites with interfacial displacement and traction discontinuities. Int. J. Solids Struct 51, 2283-2296.

Gurtin, M.E., Murdoch, A.I., 1975. A continuum theory of elastic material surfaces. Arch. Ration. Mech. Anal. 57, $291-323$.

Gurtin, M.E., Weissmüller, J., Larche, F., 1998. A general theory of curved deformable interfaces in solids at equilibrium. Philos. Mag. A 78, 1093-1109.

Hashin, Z., 1990. Thermoelastic properties of fiber composites with imperfect interface. Mech. Mater. 8 (4), $333-348$.

Hashin, Z., 1991. Thermoelastic properties of particulate composites with imperfect interface. J. Mech. Phys. Solids 39 (6), $745-762$

Hashin, Z., 1992. Extremum principles for elastic heterogeneous media with imperfect interfaces and their application to bounding of effective moduli. J. Mech. Phys. Solids 40, 767-781.

Hashin, Z., 2002. Thin interphase/imperfect interface in elasticity with application to coated fiber composites. J. Mech. Phys. Solids 50, $2509-2537$.

Hashin, Z., Rosen, B.W., 1964. The elastic moduli of fiber-reinforced materials. J. Appl. Mech. 31, 223-232.

He, J., Lilley, C.M., 2008. Surface effect on the elastic behavior of static bending nanowires. Nano Lett. 8 (7), 1798-1802.

Hill, R., 1963. Elastic properties of reinforced solids: some theoretical principles. J. Mech. Phys. Solids 11, 357-372.

Hill, R., 1972. On constitutive macro-variables for heterogeneous solids at finite strain. Proc. R. Soc. A 326, 131-147.

Huang, Z.P., Sun, L., 2007. Size-dependent effective properties of a heterogeneous material with interface energy effect: from finite deformation theory to infinitesimal strain analysis. Acta Mech. 190, 151-163.

Huang, Z.P., Wang, J., 2006. A theory of hyperelasticity of multi-phase media with surface/interface energy effect. Acta Mech. 182, 195-210.

Huang, Z.P., Wang, J., 2010. Erratum to: a theory of hyperelasticity of multi-phase media with surface/interface energy effect. Acta Mech. 215, 365-366.

Huang, Z.P., Wang, J., 2013. Micromechanics of nanocomposites with interface energy effect. In: Handbook of Micromechanics and Nanomechanics. Pan Stanford Publishing, pp. 303-348.

Javili, A., Chatzigeorgiou, G., McBride, A., Steinmann, P., Linder, C., 2015. Computational homogenization of nano-materials accounting for size effects via surface elasticity. GAMM Mitteilungen 38, 285-312.

Javili, A., Dell'Isola, F., Steinmann, P., 2013. Geometrically nonlinear higher-gradient elasticity with energetic boundaries. J. Mech. Phys. Solids 61, $2381-2401$.

Javili, A., Mcbride, A., Mergheim, J., Steinmann, P., Schmidt, U., 2013. Micro-to-macro transitions for continua with surface structure at the microscale. Int. J. Solids Struct 50, 2561-2572.

Javili, A., Mcbride, A., Steinmann, P., 2013. Thermomechanics of solids with lower-dimensional energetics: on the importance of surface, interface, and curve structures at the nanoscale. a unifying review. Appl. Mech. Rev. 65, 010802.

Javili, A., Ottosen, N. S., Ristinmaa, M., Mosler, J.,. Aspects of interface elasticity theory. Mathematics and Mechanics of Solids. doi:10.1177/1081286517699041.

Javili, A., Steinmann, P., Mosler, J., 2017. Micro-to-macro transition accounting for general imperfect interfaces. Comput. Methods Appl. Mech. Eng. 317, $274-317$.

Kanouté, P., Boso, D.P., Chaboche, J.L., Schrefler, B.A., 2009. Multiscale methods for composites: a review. Arch. Comput. Methods Eng. 16, 31-75.

Khisaeva, Z.F., Ostoja-Starzewski, M., 2006. On the size of RVE in finite elasticity of random composites. J. Elast. 85 (2), $153-173$.

Kouznetsova, V., Geers, M., Brekelmans, W., 2004. Multi-scale second-order computational homogenization of multi-phase materials: a nested finite element solution strategy. Comput. Methods Appl. Mech. Eng. 193, 5525-5550.

Kouznetsova, V., Geers, M.G.D., Brekelmans, W.A.M., 2002. Multi-scale constitutive modelling of heterogeneous materials with a gradient-enhanced computational homogenization scheme. Int. J. Numer. Methods Eng. 54, 1235-1260.

Levitas, V.I., Samani, K., 2011. Size and mechanics effects in surface-induced melting of nanoparticles.. Nature communications 2, 284.

Li, Y., Waas, A.M., Arruda, E.M., 2011. A closed-form, hierarchical, multi-interphase model for composites - derivation, verification and application to nanocomposites. J. Mech. Phys. Solids 59, 43-63.

Lim, C.W., Li, Z.R., He, L.H., 2006. Size dependent, non-uniform elastic field inside a nano-scale spherical inclusion due to interface stress. Int. J. Solids Struct 43, 5055-5065.

Liu, L., Yu, M., Lin, H., Foty, R., 2017. Deformation and relaxation of an incompressible viscoelastic body with surface viscoelasticity. J. Mech. Phys. Solids 98, 309-329.

Matous, K., Geers, M.G., Kouznetsova, V.G., Gillman, A., 2017. A review of predictive nonlinear theories for multiscale modeling of heterogeneous materials. J. Comput. Phys. 330, 192-220.

McBride, A., Mergheim, J., Javili, A., Steinmann, P., Bargmann, S., 2012. Micro-to-macro transitions for heterogeneous material layers accounting for in-plane stretch. J. Mech. Phys. Solids 60, 1221-1239.

Miller, R.E., Shenoy, V.B., B., V., 2000. Size-dependent elastic properties of nanosized structural elements. Nanotechnology 11, 139-147.

Moeckel, G.P., 1975. Thermodynamics of an interface. Arch. Ration. Mech. Anal. 57, 255-280.

Mogilevskaya, S.G., Crouch, S.L., Stolarski, H.K., 2008. Multiple interacting circular nano-inhomogeneities with surface/interface effects. J. Mech. Phys. Solids 56, 2298-2327.

Monchiet, V., Bonnet, G., 2010. Interfacial models in viscoplastic composites materials. Int. J. Eng. Sci. 48, 1762-1768.

Monteiro, E., He, Q.C., Yvonnet, J., 2011. Hyperelastic large deformations of two-phase composites with membrane-type interface. Int. J. Eng. Sci. 49, 985-1000.

Mosler, J., Scheider, I., 2011. A thermodynamically and variationally consistent class of damage-type cohesive models. J. Mech. Phys. Solids 59, 1647-1668.

Mura, T., Shodja, H.M., Hirose, Y., 1996. Inclusion problems. Appl. Mech. Rev. 49, 118-127.

Murdoch, A.I., 1976. A thermodynamical theory of elastic material interfaces. Q. J. Mech. Appl. Math. 29, $245-275$.

Nazarenko, L., Bargmann, S., Stolarski, H., 2017. Closed-form formulas for the effective properties of random particulate nanocomposites with complete Gurtinmurdoch model of material surfaces. Continuum Mech. Thermodyn. 29, 77-96.

Ogden, R., 1974. On the overall moduli of non-linear elastic composite materials. J. Mech. Phys. Solids 22, 541-553.

Olsson, P.A.T., Park, H.S., 2012. On the importance of surface elastic contributions to the flexural rigidity of nanowires. J. Mech. Phys. Solids 60 (12), 2064-2083.

Ortiz, M., Pandolfi, A., 1999. Finite-deformation irreversible cohesive elements for three-dimensional crack-propagation analysis. Int. J. Numer. Methods Eng. 44, 1267-1282.

Ostoja-Starzewski, M., Kale, S., Karimi, P., Malyarenko, A., Raghavan, B., Ranganathan, S., Zhang, J., 2016. Scaling to RVE in random media. Adv. Appl. Mech. 49, 111-211.

Park, H.S., Klein, P., 2007. Surface cauchy-born analysis of surface stress effects on metallic nanowires. Phys. Rev. B 75, 085408.

Park, H.S., Klein, P., 2008. Surface stress effects on the resonant properties of metal nanowires: the importance of finite deformation kinematics and the impact of the residual surface stress. J. Mech. Phys. Solids 56, 3144-3166.

Park, H.S., Klein, P., Wagner, G., 2006. A surface cauchy-born model for nanoscale materials. Int. J. Numer. Methods Eng. 68, 1072-1095.

Park, K., Paulino, G.H., 2013. Cohesive zone models: a critical review of traction-separation relationships across fracture surfaces. Appl. Mech. Rev. 64. 060802.

Park, K., Paulino, G.H., Roesler, J.R., 2009. A unified potential-based cohesive model of mixed-mode fracture. J. Mech. Phys. Solids 57, $891-908$.

Qian, J., Lin, J., Xu, G.-K., Lin, Y., Gao, H., 2017. Thermally assisted peeling of an elastic strip in adhesion with a substrate via molecular bonds. J. Mech. Phys. Solids 101, 197-208.

Saeb, S., Steinmann, P., Javili, A., 2016. Aspects of computational homogenization at finite deformations: a unifying review from Reuss' to Voigt's bound. Appl. Mech. Rev. 68. 050801

Sharma, P., 2004. Size-dependent elastic fields of embedded inclusions in isotropic chiral solids. Int. J. Solids Struct 41, 6317-6333.

Sharma, P., Ganti, S., 2004. Size-dependent Eshelby's tensor for embedded nano-inclusions incorporating surface / interface energies. J. Appl. Mech. 71, 663-671. 
Sharma, P., Ganti, S., Bhate, N., 2003. Effect of surfaces on the size-dependent elastic state of nano-inhomogeneities. Appl. Phys. Lett. 82, 535-537. Sharma, P., Wheeler, L.T., 2007. Size-dependent elastic state of ellipsoidal nano-inclusions incorporating surfaceinterface tension. J. Appl. Mech. 74 . 447. Steigmann, D.J., Ogden, R.W., 1999. Elastic surface-substrate interactions. Proc. R. Soc. A 455, 437-474.

Steinmann, P., 2008. On boundary potential energies in deformational and configurational mechanics. J. Mech. Phys. Solids 56, $772-800$.

Tian, L., Rajapakse, R.K.N.D., 2007. Analytical solution for size-dependent elastic field of a nanoscale circular inhomogeneity. J. Appl. Mech. 74, 568-574.

Tijssens, M.G.A., Sluys, B.L.J., Giessen, E.V.d., 2000. Numerical simulation of quasi-brittle fracture using damaging cohesive surfaces. Eur. J. Mech. A. Solids 19, 761-779.

Tu, W., Pindera, M.-J., 2014. Cohesive zone-based damage evolution in periodic materials via finite-volume homogenization. J. Appl. Mech. 81, 101005.

Wang, J., Duan, H.L., Zhang, Z., Huang, Z.P., 2005. An anti-interpenetration model and connections between interphase and interface models in particle-reinforced composites. Int. J. Mech. Sci. 47, 701-718.

Wang, Y., Weissmüller, J., Duan, H.L., 2010. Mechanics of corrugated surfaces. J. Mech. Phys. Solids 58, 1552-1566.

Wang, Z.Q., Zhao, Y.P., Huang, Z.P., 2010. The effects of surface tension on the elastic properties of nano structures. Int. J. Eng. Sci. 48, $140-150$.

Wu, C., Gowrishankar, S., Huang, R., Liechti, K.M., 2016. On determining mixed-mode traction-separation relations for interfaces. Int. J. Fract. 202 , 1-19.

Xu, X.-P., Needleman, A., 1994. Numerical simulations of fast crack growth in brittle solids. J. Mech. Phys. Solids 42, $1397-1434$.

Yvonnet, J., Mitrushchenkov, A., Chambaud, G., He, Q. C., Gu, S. T.,. Characterization of surface and nonlinear elasticity in wurtzite ZnO nanowires. J. Appl. Phys. 111 (12).

Yvonnet, J., Quang, H.L., He, Q.C., 2008. An XFEM/level set approach to modelling surface/interface effects and to computing the size-dependent effective properties of nanocomposites. Comput. Mech. 42, 119-131. 